\title{
THE MOMENTS OF THE RIEMANN ZETA-FUNCTION PART I: THE FOURTH MOMENT OFF THE CRITICAL LINE
}

\author{
Aleksandar Ivić \& Yoichi Motohashi
}

Dedicated to Professor Eduard Wirsing on the occasion of his 75 th birthday

Abstract: In this paper, the first part of a larger work, we prove the spectral decomposition of

$$
\int_{-\infty}^{\infty}|\zeta(\sigma+i t)|^{4} g(t) \mathrm{d} t \quad\left(\frac{1}{2}<\sigma<1 \text { fixed }\right)
$$

where $g(t)$ is a suitable weight function of fast decay. This is used to obtain estimates and omega results for the function

$$
\begin{aligned}
E_{2}(T, \sigma):= & \int_{0}^{T}|\zeta(\sigma+i t)|^{4} \mathrm{~d} t-\frac{\zeta^{4}(2 \sigma)}{\zeta(4 \sigma)} T-\frac{T}{3-4 \sigma}\left(\frac{T}{2 \pi}\right)^{2-4 \sigma} \frac{\zeta^{4}(2-2 \sigma)}{\zeta(4-4 \sigma)} \\
& -T^{2-2 \sigma}\left(a_{0}(\sigma)+a_{1}(\sigma) \log T+a_{2}(\sigma) \log ^{2} T\right),
\end{aligned}
$$

the error term in the asymptotic formula for the fourth moment of $|\zeta(\sigma+i t)|$.

Keywords: Fourth moment of the Riemann zeta-function, spectral decomposition, Hecke series, hypergeometric function, omega results.

\section{Introduction}

Power moments of the Riemann zeta-function $\zeta(s)$ are one of the central objects in the theory of $\zeta(s)$, with many important applications. Although the main interest is in the moments on the "critical line" $\operatorname{Re} s=\frac{1}{2}$, the moments when $s$ lies in the "critical strip" $\frac{1}{2}<\operatorname{Re} s<1$, or "off" the critical line, are also of great interest. There exist extensive results on the second and fourth moments on the critical line, the only ones that so far can be treated unconditionally, and where asymptotic formulas have been obtained. A comprehensive review on mean square results for $\zeta(s)$ is given by Matsumoto [Ma], where further references may be

2001 Mathematics Subject Classification: 11M06, 11F72, 11F66. 
found. Some of the relevant works on the fourth moment of $\left|\zeta\left(\frac{1}{2}+i t\right)\right|$ are [I1], [I2], [I4]- [I8], [IM1]- [IM3], [IJM], [Mo2]- [Mo6], where also the interested reader may find further references. The aim of this paper is to treat the fourth moment off the critical line. The only works that seem to have explicitly dealt with this subject are [I6], [K1] and [K2]. Thus it appears that the time is ripe for an extensive account of this subject, which we hope that the present work will provide.

The main object of our study is the weighted integral

$$
\mathcal{L}(g ; \sigma, \tau):=\int_{-\infty}^{\infty}|\zeta(\sigma+i t)|^{2}|\zeta(\tau+i t)|^{2} g(t) \mathrm{d} t,
$$

where $\sigma, \tau$ are given constants satisfying

$$
\frac{1}{2} \leqslant \sigma \leqslant \tau \quad(\sigma \neq 1, \tau \neq 1) .
$$

The basic assumption on the weight $g$ is: The even function $g(t)$ takes real values on the real axis, and there exists a large positive constant $A$ such that $g(t)$ is regular and $g(t)=O\left((|t|+1)^{-A}\right)$ in the horizontal strip $|\operatorname{Im} t| \leqslant A$.

We shall obtain the spectral decomposition of $\mathcal{L}(g ; \sigma, \tau)$ (see Section 3 ) by the method used by the second author in the case of $\mathcal{L}\left(g ; \frac{1}{2}, \frac{1}{2}\right)$ (see [Mo2], [Mo6]). This decomposition, which is in fact an exact identity, will contain, among other things, the function

$$
g^{*}(\xi)=\int_{-\infty}^{\infty} g(t) \mathrm{e}^{-i \xi t} \mathrm{~d} t \quad(\xi \in \mathbb{R}),
$$

namely the Fourier transform of $g$. Note that, since $g$ is even,

$$
g^{*}(\xi)=\int_{-\infty}^{\infty} g(t) \mathrm{e}^{i \xi t} \mathrm{~d} t=\int_{-\infty}^{\infty} g(t) \cos (\xi t) \mathrm{d} t=g_{c}(\xi),
$$

where $g_{c}$ is the cosine Fourier transform of $g$. The function $\mathcal{L}(g ; \sigma, \tau)$ is, with an appropriate choice of the weight $g$, the local object which after the integration over a suitable parameter contained in $g$ will lead to the asymptotic evaluation of the global object

$$
\int_{0}^{T}|\zeta(\sigma+i t)|^{2}|\zeta(\tau+i t)|^{2} \mathrm{~d} t
$$

provided that (1.2) holds. A good choice of $g$ will entail rapid decay of $g_{c}$, which will facilitate handling of the quantities that will appear in the spectral decomposition.

It is clear that (1.5) is not interesting when $\sigma>1, \tau>1$, in which case the zeta-values in question are represented by absolutely convergent series which may be readily integrated termwise. The special cases of interest of (1.1) and (1.5) are 
a) $\sigma=\tau=\frac{1}{2}$. This is the classical case of the fourth moment of $\zeta(s)$ on the critical line, and probably the most important case. The explicit formula by the second author [Mo2], and is extensively discussed in [I2] and [Mo6].

b) $\frac{1}{2}<\sigma=\tau<1$. This is the case of the fourth moment of $\zeta(s)$ off the critical line. As already mentioned, this is discussed by the first author in [I6] and by A. Kačènas [K1], [K2]. The formula for the fourth moment reads (when $\frac{1}{2}<\sigma<1$ is fixed)

$$
\begin{aligned}
\int_{0}^{T}|\zeta(\sigma+i t)|^{4} \mathrm{~d} t= & \frac{\zeta^{4}(2 \sigma)}{\zeta(4 \sigma)} T+\frac{T}{3-4 \sigma}\left(\frac{T}{2 \pi}\right)^{2-4 \sigma} \frac{\zeta^{4}(2-2 \sigma)}{\zeta(4-4 \sigma)} \\
& +T^{2-2 \sigma}\left(a_{0}(\sigma)+a_{1}(\sigma) \log T+a_{2}(\sigma) \log ^{2} T\right)+E_{2}(T, \sigma)
\end{aligned}
$$

where $E_{2}(T, \sigma)$ is the error term, and the $a_{j}(\sigma)$ 's are constants which may be explicitly evaluated. When $\sigma \rightarrow \frac{1}{2}+0$ the function $E_{2}(T, \sigma)$ tends to $E_{2}\left(T, \frac{1}{2}\right) \equiv$ $E_{2}(T)$, the error term in the asymptotic formula for $\int_{0}^{T}\left|\zeta\left(\frac{1}{2}+i t\right)\right|^{4} \mathrm{~d} t$.

In [I6] only a sketch of the spectral decomposition of the fourth moment off the critical line, due to the second author, was given. Here we are going to give a rigorous proof of the spectral decomposition in question and to recover and extend the results given in [I6]. The works of Kačènas contain an explicit evaluation of the main term in the asymptotic formulas for the fourth moment off the critical line, but the estimates for the error term are weaker than those given in [I6]. We also remark that the cases a) and b) have their analogues (mean squares) for automorphic $L$-functions (see [Mo3], [Mo7]). The fourth moment of $\zeta(s)$ off the critical line has its analogue in the mean square off the critical line. In this case, which is less difficult to deal with than the present case, the formula reads

$$
\begin{array}{r}
\int_{0}^{T}|\zeta(\sigma+i t)|^{2} \mathrm{~d} t=\zeta(2 \sigma) T+(2 \pi)^{2 \sigma-1} \frac{\zeta(2-2 \sigma)}{2-2 \sigma} T^{2-2 \sigma}+E_{1}(T, \sigma) \\
\left(\frac{1}{2}<\sigma<1\right),
\end{array}
$$

where $E_{1}(T, \sigma)$ in (1.7) represents the error term, and the notational analogy between $E_{1}(T, \sigma)$ and $E_{2}(T, \sigma)$ is obvious. As we already mentioned, [Ma] represents a comprehensive survey of results on $E_{1}(T, \sigma)$.

c) $\sigma=\frac{1}{2}, \frac{1}{2}<\tau<1$. This case, which does not seeem to have been treated in the literature before, may be thought of as a "hybrid mean value".

d) $\sigma=\frac{1}{2}, \tau>1$. This case is an extension of c). When $\tau$ is large, it is of interest because then $\mathcal{L}\left(g ; \frac{1}{2}, \tau\right)$ is bounded by of

$$
\int_{-\infty}^{\infty}\left|\zeta\left(\frac{1}{2}+i t\right)\right|^{2} g(t) \mathrm{d} t
$$

and provides the mean (at least theoretically) to estimate $\zeta\left(\frac{1}{2}+i t\right)$ pointwise, which is a fundamental problem in the theory of $\zeta(s)$. 
In this paper, which is Part I of the whole work, we shall treat the case b) above. To avoid excessive length, the cases c) and d) will be treated in Part II. The plan of the present paper is as follows. The formulation of the spectral decomposition of $\mathcal{L}(g ; \tau, \tau)$, when $\frac{1}{2}<\tau<1$, will be given in Section 2 . Although the proof has many analogies with the proof of the second author for the case of $\mathcal{L}\left(g ; \frac{1}{2}, \frac{1}{2}\right)$, there are also many detours, and the complete, rigorous proof is given in Section 3. In the result $\alpha_{j} H_{j}^{2}\left(\frac{1}{2}\right) H_{j}(\tau)$ and $\alpha_{j} H_{j}\left(\frac{1}{2}\right) H_{j}^{2}(\tau)$ (in Part II) appear, and the asymptotic evaluation of sums of these quantities over $\kappa_{j} \leqslant K$ is carried out in Section 4. The detailed asymptotic evaluation of the function $\Lambda$, appearing in the spectral decomposition of $\mathcal{L}(g ; \tau, \tau)$ with the Gaussian weight function, is contained in Section 5. The explicit formulas for $\mathcal{L}(g ; \tau, \tau)$ and its integral are presented in Section 6. They are necessary in order to obtain results on the error term $E_{2}(T, \sigma)$, which is done in Section 7 and Section 8. The notation used throughout the paper is, whenever possible, standard. We have used the letter $\tau$ occasionally where one would commonly used $\sigma$ (as in the notation for $E_{2}(T, \sigma)$ ). This was done to avoid possible confusion with the real part of the complex variable $s$, especially in Section 3 .

\section{Spectral decomposition of the fourth moment - notation and results}

In this section we introduce the necessary notation for the spectral decomposition of $\mathcal{L}(g ; \tau, \tau)$, the weighted fourth moment off the critical line. We also present Theorem 1, which will give the desired decomposition, but postpone the proof for Section 3. The notation used throughout is standard, to be found e.g., in the second author's monograph [Mo6], and for this reason we shall be relatively brief.

Let $\left\{\lambda_{j}=\kappa_{j}^{2}+\frac{1}{4}\right\} \cup\{0\}$ be the discrete spectrum of the hyperbolic Laplacian

$$
\Delta=-y^{2}\left(\left(\frac{\partial}{\partial x}\right)^{2}+\left(\frac{\partial}{\partial y}\right)^{2}\right)
$$

acting over the Hilbert space composed of all $\Gamma$-automorphic functions which are square integrable with respect to the hyperbolic measure, where

$$
\Gamma \cong \operatorname{SL}(2, \mathbb{Z}) /\{+1,-1\}
$$

Let $\left\{\psi_{j}\right\}$ be a maximal orthonormal system in this space such that $\Delta \psi_{j}=\lambda_{j} \psi_{j}$ for each $j \geqslant 1$ and $T(n) \psi_{j}=t_{j}(n) \psi_{j}$ for each integer $n \in \mathbb{N}$, where

$$
(T(n) f)(z)=\frac{1}{\sqrt{n}} \sum_{a d=n} \sum_{b=1}^{d} f\left(\frac{a z+b}{d}\right)
$$


is the Hecke operator. We shall further assume that $\psi_{j}(-\bar{z})=\epsilon_{j} \psi_{j}(z)$ with the parity sign $\epsilon_{j}= \pm 1$. We then define ( $s=\sigma+i t$ will denote a complex variable)

$$
H_{j}(s)=\sum_{n=1}^{\infty} t_{j}(n) n^{-s} \quad(\sigma>1)
$$

which denotes the Hecke series associated with $\psi_{j}(z)$, and which can be continued analytically to an entire function. As usual we put

$$
\alpha_{j}=\left|\rho_{j}(1)\right|^{2}\left(\cosh \pi \kappa_{j}\right)^{-1},
$$

where $\rho_{j}(1)$ is the first Fourier coefficient of $\psi_{j}(z)$. The holomorphic counterparts $\alpha_{j, k}$ and

$$
H_{j, k}(s):=\sum_{n=1}^{\infty} t_{j, k}(n) n^{-s} \quad(\operatorname{Re} s>1)
$$

of (2.2) and (2.1), respectively, are defined in [Mo6, Chapter 3]; as to $\vartheta(2 k)$ in (2.7) below see Section 2.2 there. Now we can formulate

Theorem 1. Let $\frac{1}{2}<\tau<1$ be fixed, and let $g$ satisfy the basic assumption. Then we have ( $c f .(1.1))$

$$
\mathcal{L}(g ; \tau, \tau)=\left\{z_{r}+z_{d}+z_{c}+z_{h}\right\}(\tau, g),
$$

where

$$
\begin{aligned}
z_{r}(\tau, g)= & M\left(\mathrm{p}_{\tau} ; g\right) \\
& -8 \pi \zeta(2 \tau-1)^{2} \operatorname{Re}\left\{\left(c_{E}-\frac{\zeta^{\prime}}{\zeta}(2 \tau-1)\right) g((\tau-1) i)+\frac{1}{2} i g^{\prime}((\tau-1) i)\right\}
\end{aligned}
$$

with the function $M$ being defined by (3.65), (3.88), and (3.92) according as $\frac{1}{2}<\tau<\frac{3}{4}, \tau=\frac{3}{4}$, and $\frac{3}{4}<\tau<1$, respectively. Further we have

$$
\begin{aligned}
& z_{d}(\tau, g)=\sum_{j=1}^{\infty} \alpha_{j} H_{j}^{2}\left(\frac{1}{2}\right) H_{j}\left(2 \tau-\frac{1}{2}\right) \Lambda\left(\kappa_{j} ; \tau, g\right), \\
& z_{c}(\tau, g)=\frac{1}{\pi} \int_{-\infty}^{\infty} \frac{\left|\zeta\left(\frac{1}{2}+i r\right)\right|^{4}\left|\zeta\left(2 \tau-\frac{1}{2}+i r\right)\right|^{2}}{|\zeta(1+2 i r)|^{2}} \Lambda(r ; \tau, g) \mathrm{d} r, \\
& z_{h}(\tau, g)=\sum_{k=1}^{\infty} \sum_{j=1}^{\vartheta(2 k)} \alpha_{j, 2 k} H_{j, 2 k}^{2}\left(\frac{1}{2}\right) H_{j, 2 k}\left(2 \tau-\frac{1}{2}\right) \Lambda\left(\left(\frac{1}{2}-2 k\right) i ; \tau, g\right) .
\end{aligned}
$$

Here $c_{E}=-\Gamma^{\prime}(1)$ is Euler's constant, and

$$
\begin{aligned}
& \Lambda(r ; \tau, g)=\int_{0}^{\infty}(y(1+y))^{-\tau} g_{c}\left(\log \left(1+\frac{1}{y}\right)\right) \\
& \quad \times \operatorname{Re}\left\{y^{-\frac{1}{2}-i r}\left(1+\frac{i}{\sinh (\pi r)}\right) \frac{\Gamma\left(\frac{1}{2}+i r\right)^{2}}{\Gamma(1+2 i r)} F\left(\frac{1}{2}+i r, \frac{1}{2}+i r ; 1+2 i r ;-\frac{1}{y}\right)\right\} \mathrm{d} y
\end{aligned}
$$

with the hypergeometric function $F$. 
The above spectral decomposition is analogous to the spectral decomposition of the function $\mathcal{L}\left(g ; \frac{1}{2}, \frac{1}{2}\right)$, given as [Mo6, Theorem 4.2]. It is in fact an exact identity, relating the original object (weighted integral of the fourth moment) to various objects from spectral theory, hence the terminology "spectral decomposition". The notation is also analogous to the one used in [Mo6, Theorem 4.2], as much as possible. The notation $M\left(\mathrm{p}_{\tau} ; g\right)$ refers to the "main term", since suitable integration of this term will lead to the main term for the fourth moment of $|\zeta(\sigma+i t)|$ itself (see (1.6)). Likewise, the notation $z_{r}, z_{d}, z_{c}, z_{h}$ refers to "residual", "discrete", "continuous" and "holomorphic" parts, respectively. As we just mentioned, the term $M\left(\mathrm{p}_{\tau} ; g\right)$, contained in $\mathcal{z}_{r}$, will eventually contribute to the main term, while the remaining terms will contribute to the error terms. Of these, the most difficult (major) contribution, like in the case of $\mathcal{L}\left(g ; \frac{1}{2}, \frac{1}{2}\right)$, will come from $z_{d}$.

An important feature of the above formula is the appearance of the oscillatory integral $\Lambda(r ; \tau, g)$ which containins the hypergeometic function. We recall here that, for $|z|<1$, one defines the hypergeometric function

$$
\begin{aligned}
F(\alpha, \beta ; \gamma ; z) & =\sum_{k=0}^{\infty} \frac{(\alpha)_{k}(\beta)_{k}}{(\gamma)_{k} k !} z^{k} \\
& =1+\sum_{k=1}^{\infty} \frac{\alpha(\alpha+1) \ldots(\alpha+k-1) \beta(\beta+1) \ldots(\beta+k-1)}{\gamma(\gamma+1) \ldots(\gamma+k-1) k !} z^{k} .
\end{aligned}
$$

Analytic continuation and other properties of $F(\alpha, \beta ; \gamma ; z)$ are treated e.g., by N.N. Lebedev [L].

\section{Proof of the spectral decomposition for the fourth moment}

This section contains the proof of Theorem 1; we assume throughout that $\frac{1}{2}<\tau<$ 1 is fixed, and that the basic assumption on $g$ holds. Our argument is a reworking of [Mo6, Chapter 4]; thus we could mention specific changes only. However, that would make the later part of our discussion hard to comprehend, since as has been mentioned above there are many sensitive detours peculiar to our new situation that begins in fact at (3.7) below.

Let first

$$
\begin{aligned}
\tilde{g}(s, \lambda) & =\int_{0}^{\infty} y^{s-1}(1+y)^{-\lambda} g^{*}(\log (1+y)) \mathrm{d} y \\
& =\Gamma(s) \int_{-\infty+A i}^{\infty+A i} \frac{\Gamma(\lambda-i t-s)}{\Gamma(\lambda-i t)} g(t) \mathrm{d} t,
\end{aligned}
$$

where $g^{*}$ is defined by (1.3). We begin with the analogue of [Mo6, Lemma 4.1], namely 
Lemma 1. The function $\tilde{g}(s, \lambda) / \Gamma(s)$ continues holomorphically to the domain

$$
|\operatorname{Re} s| \leqslant \frac{1}{3} A, \quad|\operatorname{Re} \lambda| \leqslant \frac{1}{3} A
$$

and there we have

$$
\tilde{g}(s, \lambda) \ll|s|^{-\frac{1}{2} A},
$$

when $s$ tends to infinity while $\lambda$ remains bounded.

Let now $\mathcal{D}_{+}$and $\mathcal{D}_{-}$be the domains of $\mathbb{C}^{4}$ where all four variables have real parts larger than and less than one, respectively. We set, for $(u, v, w, z) \in \mathcal{D}_{+}$,

$$
\mathcal{J}(u, v, w, z ; g):=\int_{-\infty}^{\infty} \zeta(u+i t) \zeta(v+i t) \zeta(w-i t) \zeta(z-i t) g(t) \mathrm{d} t
$$

Moving the path upwards appropriately, we see that $\mathcal{J}$ is a meromorphic function over the domain

$$
\mathcal{B}=\left\{(u, v, w, z) \in \mathbb{C}^{4}:|u|,|v|,|w|,|z|<B\right\}
$$

where $B=c A$ with $0<c<1$ is supposed to be sufficiently large. Then, taking $(u, v, w, z)$ in $\mathcal{D}_{-} \cap \mathcal{B}$, we get the following meromorphic continuation of $\mathcal{J}$ to $\mathcal{D}_{-} \cap \mathcal{B}:$

$$
\begin{aligned}
\mathcal{J}(u, v, w, z ; g)= & \int_{-\infty}^{\infty} \zeta(u+i t) \zeta(v+i t) \zeta(w-i t) \zeta(z-i t) g(t) \mathrm{d} t \\
& +2 \pi \zeta(v-u+1) \zeta(u+w-1) \zeta(u+z-1) g((u-1) i) \\
& +2 \pi \zeta(u-v+1) \zeta(v+w-1) \zeta(v+z-1) g((v-1) i) \\
& +2 \pi \zeta(z-w+1) \zeta(u+w-1) \zeta(v+w-1) g((1-w) i) \\
& +2 \pi \zeta(w-z+1) \zeta(u+z-1) \zeta(v+z-1) g((1-z) i) .
\end{aligned}
$$

Lemma 2. The function $\mathcal{J}$ is regular at the point $\mathrm{p}_{\tau}:=(\tau, \tau, \tau, \tau)$, and we have

$$
\begin{aligned}
& \mathcal{L}(g ; \tau, \tau)=\mathcal{J}\left(\mathrm{p}_{\tau} ; g\right) \\
& -8 \pi \zeta(2 \tau-1)^{2} \operatorname{Re}\left\{\left(c_{E}-\frac{\zeta^{\prime}}{\zeta}(2 \tau-1)\right) g((\tau-1) i)+\frac{1}{2} i g^{\prime}((\tau-1) i)\right\},
\end{aligned}
$$

where $c_{E}=-\Gamma^{\prime}(1)$ is Euler's constant.

Proof. On the right side of (3.6) the integral is obviously regular throughout $\mathcal{D}_{-} \cap \mathcal{B}$. To see the regularity at $\mathrm{p}_{\tau}$ of the sum of other terms, we need only to 
replace the factors $\zeta(v-u+1), \zeta(u-v+1), \zeta(z-w+1)$ and $\zeta(w-z+1)$ by their Laurent expansions. For example, its value at $\mathrm{p}_{\tau}$ is

$$
\begin{aligned}
& 4 \pi \zeta(2 \tau-1)^{2} c_{E}\{g((\tau-1) i)+g((1-\tau) i)\} \\
& \quad-2 \pi\left[\frac{\partial}{\partial v}\{\zeta(v+w-1) \zeta(v+z-1) g((v-1) i)\}\right]_{\mathrm{p}_{\tau}} \\
& \quad-2 \pi\left[\frac{\partial}{\partial z}\{\zeta(u+z-1) \zeta(v+z-1) g((1-z) i)\}\right]_{\mathrm{p}_{\tau}} \\
& =4 \pi\left\{\zeta(2 \tau-1)^{2} c_{E}-\zeta(2 \tau-1) \zeta^{\prime}(2 \tau-1)\right\}\{g((\tau-1) i)+g((1-\tau) i)\} \\
& \quad+2 \pi \zeta(2 \tau-1)^{2} i\left\{g^{\prime}((\tau-1) i)-g^{\prime}((1-\tau) i)\right\} .
\end{aligned}
$$

Next, in $\mathcal{D}_{+}$we have

$$
\begin{aligned}
\mathcal{J}(u, v, w, z ; g) & =\sum_{k, l, m, n=1}^{\infty} k^{-u} l^{-v} m^{-w} n^{-z} g^{*}(\log (m n) /(k l)) \\
& =\mathcal{J}_{0}(u, v, w, z ; g)+\mathcal{J}_{1}(u, v, w, z ; g)+\overline{\partial_{1}(\bar{w}, \bar{z}, \bar{u}, \bar{v} ; g)},
\end{aligned}
$$

where $\mathfrak{J}_{0}$ and $\mathcal{J}_{1}$ correspond to the parts with $k l=m n$ and $k l<m n$, respectively. We have

$$
\jmath_{0}(u, v, w, z ; g)=g^{*}(0) \zeta(u+w) \zeta(u+z) \zeta(v+w) \zeta(v+z) / \zeta(u+v+w+z),
$$

and

$$
\partial_{1}(u, v, w, z ; g)=\frac{1}{2 \pi i} \sum_{m, n=1}^{\infty} \frac{\sigma_{u-v}(m) \sigma_{w-z}(m+n)}{m^{u+w}} \int_{(2)} \tilde{g}(s, w)(m / n)^{s} \mathrm{~d} s
$$

where $\int_{(c)} \cdots \mathrm{d} s$ denotes integration over the line $\operatorname{Re} s=c$, and $\sigma_{a}(n)=\sum_{d \mid n} d^{a}$. One may deal with this double sum in two ways: either by using the Ramanujan expansion of the function $\sigma_{w-z}(m+n)$, or by embedding $\mathcal{J}_{1}$ in values of a Poincaré series on $\Gamma \backslash \mathrm{PSL}_{2}(\mathbb{R})$. Here the first method is employed, and we shall follow [Mo6, Chapter 4]. As to the second method, see [BM]. It should be remarked that the latter dispenses with the spectral theory of sums of Kloosterman sums that plays a predominant rôle in the former. Also it should be added in this context that Theorem 1 above could be formulated solely in terms of the $\Gamma$-automorphic representations of $\mathrm{PSL}_{2}(\mathbb{R})$.

Lemma 3. The function $\mathfrak{J}_{1}(u, v, w, z ; g)$ can be continued meromorphically to the domain

$$
\begin{aligned}
\mathcal{E}:= & \left\{(u, v, w, z) \in \mathcal{B}: \operatorname{Re}(u+w)<\frac{1}{3} B, \operatorname{Re}(v+w)<\frac{1}{3} B,\right. \\
& \operatorname{Re}(u+v+w+z)>3 B\},
\end{aligned}
$$


and in $\mathcal{E}$ we have the decomposition

$$
\mathcal{J}_{1}(u, v, w, z)=\mathcal{J}_{2}(u, v, w, z)+\mathcal{J}_{3}^{+}(u, v, w, z)+\mathcal{J}_{3}^{-}(u, v, w, z) .
$$

Here

$$
\begin{aligned}
\jmath_{2}(u, v, w, z ; g):= & \tilde{g}(u+w-1) \zeta(v+z) \zeta(u+w-1) \\
& \times \zeta(z-w+1) \zeta(v-u+1) / \zeta(v+z-u-w+2), \\
& +\tilde{g}(v+w-1) \zeta(u+z) \zeta(v+w-1) \\
& \times \zeta(z-w+1) \zeta(u-v+1) / \zeta(u+z-v-w+2)
\end{aligned}
$$

and

$$
\begin{aligned}
& \mathcal{J}_{3}^{ \pm}(u, v, w, z ; g):=2(2 \pi)^{w-z-1} \zeta(z-w+1) \\
& \quad \times \sum_{m, n=1}^{\infty} m^{\frac{1}{2}(1-u-v-w-z)} n^{\frac{1}{2}(u+w-v-z-1)} \sigma_{v-u}(n) K_{ \pm}(m, n ; u, v, w, z ; g),
\end{aligned}
$$

where

$$
K_{ \pm}(m, n ; u, v, w, z ; g)=\sum_{l=1}^{\infty} \frac{1}{l} S(m, \pm n ; l) \varphi_{ \pm}\left(\frac{4 \pi}{l} \sqrt{m n} ; u, v, w, z ; g\right)
$$

with $S(a, b ; c)=\sum_{1 \leqslant n \leqslant c,(n, c)=1, n \bar{n} \equiv 1(\bmod c)} \exp \left(2 \pi i\left(\frac{a n+b \bar{n}}{c}\right)\right)$ a Kloosterman sum, and

$$
\begin{aligned}
& \varphi_{+}(x ; u, v, w, z ; g):=\frac{1}{2 \pi i} \cos \left(\frac{1}{2}(u-v) \pi\right) \int_{(B)}\left(\frac{x}{2}\right)^{u+v+w+z-1-2 s} \\
& \quad \times \Gamma(s+1-u-w) \Gamma(s+1-v-w) \tilde{g}(s, w) \mathrm{d} s \\
& \varphi_{-}(x ; u, v, w, z ; g):=-\frac{1}{2 \pi i} \int_{(B)}\left(\frac{x}{2}\right)^{u+v+w+z-1-2 s} \cos \left(\pi\left(w+\frac{1}{2}(u+v)-s\right)\right) \\
& \quad \times \Gamma(s+1-u-w) \Gamma(s+1-v-w) \tilde{g}(s, w) \mathrm{d} s
\end{aligned}
$$

The Kloosterman-Spectral sum formula of N.V. Kuznetsov (see [Mo6]) yields, with the standard notation from the spectral theory of the Fourier coefficients of modular cusp forms, that

$$
\begin{aligned}
& K_{+}(m, n ; u, v, w, z ; g):=\sum_{j=1}^{\infty} \alpha_{j} t_{j}(m) t_{j}(n)\left(\varphi_{+}\right)^{+}\left(\kappa_{j} ; u, v, w, z ; g\right) \\
& \quad+\frac{1}{\pi} \int_{-\infty}^{\infty} \frac{\sigma_{2 i r}(m) \sigma_{2 i r}(n)}{(m n)^{i r}|\zeta(1+2 i r)|^{2}}\left(\varphi_{+}\right)^{+}(r ; u, v, w, z ; g) \mathrm{d} r \\
& \quad+2 \sum_{k=1}^{\infty} \sum_{j=1}^{\vartheta(k)} \alpha_{j, k} t_{j, k}(m) t_{j, k}(n)\left(\varphi_{+}\right)^{+}\left(\left(\frac{1}{2}-k\right) i ; u, v, w, z ; g\right),
\end{aligned}
$$


where

$$
\begin{aligned}
& \left(\varphi_{+}\right)^{+}(r ; u, v, w, z ; g) \\
& :=\frac{\pi i}{2 \sinh (\pi r)} \int_{0}^{\infty}\left(J_{2 i r}(x)-J_{-2 i r}(x)\right) \varphi_{+}(x ; u, v, w, z ; g) \frac{\mathrm{d} x}{x},
\end{aligned}
$$

and $J_{\nu}(x)$ is the Bessel function of the first kind in standard notation (see $[\mathrm{L}]$ ). Also,

$$
\begin{aligned}
& K_{-}(m, n ; u, v, w, z ; g):=\sum_{j=1}^{\infty} \epsilon_{j} \alpha_{j} t_{j}(m) t_{j}(n)\left(\varphi_{-}\right)^{-}\left(\kappa_{j} ; u, v, w, z ; g\right) \\
& \quad+\frac{1}{\pi} \int_{-\infty}^{\infty} \frac{\sigma_{2 i r}(m) \sigma_{2 i r}(n)}{(m n)^{i r}|\zeta(1+2 i r)|^{2}}\left(\varphi_{-}\right)^{-}(r ; u, v, w, z ; g) \mathrm{d} r
\end{aligned}
$$

where

$$
\left(\varphi_{-}\right)^{-}(r ; u, v, w, z ; g):=2 \cosh (\pi r) \int_{0}^{\infty} \varphi_{-}(r ; u, v, w, z ; g) K_{2 i r}(x) \frac{\mathrm{d} x}{x},
$$

and $K_{\nu}(x)$ is the Bessel function of imaginary argument (or Macdonald's function).

Now, in order to facilitate later discussion, we introduce three functions $\Phi_{ \pm}$ and $\Xi$ of five complex variables:

$$
\begin{aligned}
& \Phi_{+}(\xi ; u, v, w, z ; g):=-i(2 \pi)^{w-z-2} \cos \left(\frac{1}{2} \pi(u-v)\right) \\
& \quad \times \int_{-i \infty}^{i \infty} \sin \left(\frac{1}{2} \pi(u+v+w+z-2 s)\right) \\
& \quad \times \Gamma\left(\frac{1}{2}(u+v+w+z-1)+\xi-s\right) \Gamma\left(\frac{1}{2}(u+v+w+z-1)-\xi-s\right) \\
& \quad \times \Gamma(s+1-u-w) \Gamma(s+1-v-w) \tilde{g}(s, w) \mathrm{d} s ; \\
& \Phi_{-}(\xi ; u, v, w, z ; g)=i(2 \pi)^{w-z-2} \cos (\pi \xi) \int_{-i \infty}^{i \infty} \cos \left(\pi\left(w+\frac{1}{2}(u+v)-s\right)\right) \\
& \quad \times \Gamma\left(\frac{1}{2}(u+v+w+z-1)+\xi-s\right) \Gamma\left(\frac{1}{2}(u+v+w+z-1)-\xi-s\right) \\
& \quad \times \Gamma(s+1-u-w) \Gamma(s+1-v-w) \tilde{g}(s, w) \mathrm{d} s ; \\
& \quad(\xi ; u, v, w, z ; g)=\frac{1}{2 \pi i} \int_{-\infty i}^{\infty i} \frac{\Gamma\left(\xi+\frac{1}{2}(u+v+w+z-1)-s\right)}{\Gamma\left(\xi+\frac{1}{2}(3-u-v-w-z)+s\right)} \\
& \quad \times \Gamma(s+1-u-w) \Gamma(s+1-v-w) \tilde{g}(s, w) \mathrm{d} s .
\end{aligned}
$$

Note that the path in (3.22) is such that the poles of the first two gamma-factors and those of the other three factors in the integrand are separated to the right and 
the left, respectively, by the path, and $\xi, u, v, w, z$ are assumed to be such that the path can be drawn. The path in (3.23) is chosen in just the same way. On the other hand the path in (3.24) separates the poles of $\Gamma\left(\xi+\frac{1}{2}(u+v+w+z-1)-s\right)$ and those of $\Gamma(s+1-u-w) \Gamma(s+1-v-w) \tilde{g}(s, w)$ to the left and the right of the path, respectively.

Lemma 4. We have

$$
\begin{aligned}
\Phi_{+}(\xi ; u, v, w, z ; g)= & -\frac{(2 \pi)^{w-z} \cos \left(\frac{1}{2} \pi(u-v)\right)}{4 \sin (\pi \xi)} \\
& \times\{\Xi(\xi ; u, v, w, z ; g)-\Xi(-\xi ; u, v, w, z ; g)\} \\
\Phi_{-}(\xi ; u, v, w, z ; g)= & \frac{(2 \pi)^{w-z}}{4 \sin (\pi \xi)}\left\{\sin \left(\pi\left(\frac{1}{2}(z-w)+\xi\right)\right) \Xi(\xi ; u, v, w, z ; g)\right. \\
& \left.-\sin \left(\pi\left(\frac{1}{2}(z-w)-\xi\right)\right) \Xi(-\xi ; u, v, w, z ; g)\right\},
\end{aligned}
$$

provided the left sides are well-defined. Also, for real $r$ and $(u, v, w, z) \in \mathcal{E}$ (see $(3.12))$,

$$
\begin{aligned}
& \left(\varphi_{+}\right)^{+}(r ; u, v, w, z ; g)=\frac{1}{2}(2 \pi)^{1-w+z} \Phi_{+}(i r ; u, v, w, z ; g) \\
& \left(\varphi_{-}\right)^{-}(r ; u, v, w, z ; g)=\frac{1}{2}(2 \pi)^{1-w+z} \Phi_{-}(i r ; u, v, w, z ; g)
\end{aligned}
$$

and, for integral $k \geqslant 1$ and $(u, v, w, z) \in \mathcal{E}$,

$$
\begin{aligned}
& \left(\varphi_{+}\right)^{+}\left(i\left(\frac{1}{2}-k\right) ; u, v, w, z ; g\right) \\
& \quad=\frac{1}{2}(-1)^{k} \pi \cos \left(\frac{1}{2} \pi(u-v)\right) \Xi\left(k-\frac{1}{2} ; u, v, w, z ; g\right) .
\end{aligned}
$$

The last three formulas are consequences of Mellin transforms of $J$ - and $K$ Bessel functions.

Next, we insert the spectral expansions (3.18) and (3.20) into (3.15) and exchange the order of sums and integrals. The absolute convergence that we have to check is obvious as far as the double summation over the variables $m, n$ is concerned, since we have $(u, v, w, z) \in \mathcal{E}$ and

$$
t_{j}(n) \ll n^{\frac{1}{4}+\delta}, \quad t_{j, k}(n) \ll n^{\frac{1}{4}+\delta},
$$

where the implicit constant depends only on $\delta$, an arbitrary fixed positive constant. The bounds in (3.30) are not the best ones known, but they are sufficient for our purpose. Thus the issue is reduced to bounding $\left(\varphi_{ \pm}\right)^{ \pm}$; and Lemma 4 renders it in terms of the function $\Xi$. We then have, uniformly for any fixed compact subset of $\mathcal{E}$

$$
\Xi(i r ; u, v, w, z ; g) \ll|r|^{-\frac{1}{4} A}, \quad \Xi\left(k-\frac{1}{2} ; u, v, w, z ; g\right) \ll k^{-\frac{1}{4} A},
$$


as real $r$ and positive integral $k$ tend to infinity. Hence, on noting (see [Mo6]) that

$$
\sum_{K \leqslant \kappa_{j}<2 K} \alpha_{j} \ll K^{2}, \quad \sum_{j=1}^{\vartheta(k)} \alpha_{j, k} \ll k,
$$

we are now able to exchange freely the order of sums and integrals in question, as long as we work inside $\mathcal{E}$.

Before stating our new expressions for $\mathcal{J}_{3}^{ \pm}$we put

$$
\begin{aligned}
& S(\xi ; u, v, w, z):=\zeta\left(\frac{1}{2}(u+v+w+z-1)+\xi\right) \zeta\left(\frac{1}{2}(u+v+w+z-1)-\xi\right) \\
& \quad \times \zeta\left(\frac{1}{2}(u+z-v-w+1)+\xi\right) \zeta\left(\frac{1}{2}(u+z-v-w+1)-\xi\right) \\
& \quad \times \zeta\left(\frac{1}{2}(v+z-u-w+1)+\xi\right) \zeta\left(\frac{1}{2}(v+z-u-w+1)-\xi\right) .
\end{aligned}
$$

Then we have

Lemma 5. In the domain $\mathcal{E}$ we have

$$
\mathcal{J}_{3}^{+}(u, v, w, z ; g)=\mathcal{J}_{3, c}^{+}(u, v, w, z ; g)+\mathcal{J}_{3, d}^{+}(u, v, w, z ; g)+\mathcal{J}_{3, h}^{+}(u, v, w, z ; g) \text {, }
$$

where

$$
\begin{aligned}
& \mathrm{J}_{3, c}^{+}(u, v, w, z ; g):=\frac{1}{i \pi} \int_{(0)} \frac{S(\xi ; u, v, w, z)}{\zeta(1+2 \xi) \zeta(1-2 \xi)} \Phi_{+}(\xi ; u, v, w, z ; g) \mathrm{d} \xi \\
& \mathcal{J}_{3, d}^{+}(u, v, w, z ; g) \\
& :=\sum_{j=1}^{\infty} \alpha_{j} H_{j}\left(\frac{1}{2}(u+v+w+z-1)\right) H_{j}\left(\frac{1}{2}(u+z-v-w+1)\right) \\
& \quad \times H_{j}\left(\frac{1}{2}(v+z-u-w+1)\right) \Phi_{+}\left(i \kappa_{j} ; u, v, w, z ; g\right) \\
& \mathcal{J}_{3, h}^{+}(u, v, w, z ; g):=(2 \pi)^{w-z} \cos \left(\frac{1}{2}(u-v)\right) \\
& \quad \times \sum_{k=6}^{\infty} \sum_{j=1}^{\vartheta(k)}(-1)^{k} \alpha_{j, k} H_{j, k}\left(\frac{1}{2}(u+v+w+z-1)\right) H_{j, k}\left(\frac{1}{2}(u+z-v-w+1)\right) \\
& \quad \times H_{j, k}\left(\frac{1}{2}(v+z-u-w+1)\right) \Xi\left(k-\frac{1}{2} ; u, v, w, z ; g\right) .
\end{aligned}
$$

Also

$$
\mathcal{J}_{3}^{-}(u, v, w, z ; g)=\mathcal{J}_{3, c}^{-}(u, v, w, z ; g)+\mathcal{J}_{3, d}^{-}(u, v, w, z ; g),
$$

where

$$
\begin{aligned}
& \mathcal{J}_{3, c}^{-}(u, v, w, z ; g):=\frac{1}{i \pi} \int_{(0)} \frac{S(\xi ; u, v, w, z)}{\zeta(1+2 \xi) \zeta(1-2 \xi)} \Phi_{-}(\xi ; u, v, w, z ; g) \mathrm{d} \xi \\
& \mathcal{J}_{3, d}^{-}(u, v, w, z ; g) \\
& =\sum_{j=1}^{\infty} \epsilon_{j} \alpha_{j} H_{j}\left(\frac{1}{2}(u+v+w+z-1)\right) H_{j}\left(\frac{1}{2}(u+z-v-w+1)\right) \\
& \quad \times H_{j}\left(\frac{1}{2}(v+z-u-w+1)\right) \Phi_{-}\left(i \kappa_{j} ; u, v, w, z ; g\right) .
\end{aligned}
$$


Our next task is to show that the above spectral expansions of $\mathcal{J}_{3}^{ \pm}$can be continued to the domain $\mathcal{B}$, whereby we shall finish our meromorphic continuation of $\mathcal{J}_{1}$. The domain $\mathcal{B}$ is obviously symmetric and wide enough to have a joint domain with $\mathcal{D}_{+}$, where the decomposition (3.9) was introduced. Hence (3.9) should hold throughout $\mathcal{B}$, and we shall obtain a spectral decomposition of $\mathcal{L}_{4}(g ; \tau, \tau)$, as asserted.

By virtue of Lemma 4, our problem is equivalent to studying the analytic properties of the function $\Xi$. In fact, it is meromorphic in a fairly wide domain in $\mathbb{C}^{5}$, as given by

Lemma 6. The function $\Xi(\xi ; u, v, w, z ; g)$ is meromorphic in the domain

$$
\widetilde{\mathcal{B}}=\left\{\xi: \operatorname{Re} \xi>-\frac{1}{8} A\right\} \times \mathcal{B}
$$

and regular in $\widetilde{\mathcal{B}} \backslash \mathcal{N}$, where $\mathcal{N}$ is the set of points $(\xi, u, v, w, z)$ such that at least one of

$$
\begin{aligned}
& \xi+\frac{1}{2}(u+v+w+z-1), \quad \xi+\frac{1}{2}(u+z-v-w+1), \\
& \xi+\frac{1}{2}(v+z-u-w+1)
\end{aligned}
$$

is equal to a non-positive integer. Moreover, if $|\xi|$ tends to infinity in any fixed vertical or horizontal strips while satisfying $\operatorname{Re} \xi>-\frac{1}{8} A$, then uniformly in $\mathcal{B}$ we have

$$
\Xi(\xi ; u, v, w, z ; g) \ll|\xi|^{-\frac{1}{4} A} .
$$

In passing, we record that we have also (this is [Mo6, Lemma 4.8] with $\gamma=2 \xi+1)$

Lemma 7. If $(\xi, u, v, w, z)$ is such that the path in (3.24) can be drawn in a vertical strip contained in the half plane $\operatorname{Re} s>0$, then we have

$$
\begin{aligned}
& \Xi(\xi ; u, v, w, z ; g) \\
& =\frac{\Gamma(\alpha) \Gamma(\beta)}{\Gamma(2 \xi+1)} \int_{0}^{\infty} \frac{y^{\xi+\frac{1}{2}(u+v+w+z-3)}}{(1+y)^{w}} g^{*}(\log (1+y)) F(\alpha, \beta ; 2 \xi+1 ;-y) \mathrm{d} y
\end{aligned}
$$

where $F($ see $(2.9))$ is the hypergeometric function, and

$$
\alpha=\xi+\frac{1}{2}(u+z-v-w+1), \quad \beta=\xi+\frac{1}{2}(v+z-u-w+1) .
$$

An immediate consequence of Lemma 6 is that $\mathcal{J}_{3, d}^{ \pm}$and $\mathcal{J}_{3, h}^{+}$are meromorphic inside $\mathcal{B}$. Thus, we shall consider $\mathcal{J}_{3, c}^{ \pm}$. To this end we assume first that $(u, v, w, z)$ is in $\mathcal{E}$; and put

$$
\mathcal{J}_{3, c}(u, v, w, z ; g)=\mathcal{J}_{3, c}^{+}(u, v, w, z ; g)+\mathcal{J}_{3, c}^{-}(u, v, w, z ; g) .
$$


We have, by (3.25)-(3.26),

$$
\begin{aligned}
& \partial_{3, c}(u, v, w, z ; g)=i(2 \pi)^{w-z-1} \int_{(0)} \frac{S(\xi ; u, v, w, z)}{\sin (\pi \xi) \zeta(1+2 \xi) \zeta(1-2 \xi)} \\
& \quad \times\left\{\cos \left(\frac{1}{2} \pi(u-v)\right)-\sin \left(\pi\left(\frac{1}{2}(z-w)+\xi\right)\right)\right\} \Xi(\xi ; u, v, w, z ; g) \mathrm{d} \xi .
\end{aligned}
$$

Applying the functional equation for $\zeta(s)$ to $\zeta(1-2 \xi)$, we obtain from (3.47)

$$
\begin{aligned}
& \jmath_{3, c}(u, v, w, z ; g) \\
& =2 i(2 \pi)^{w-z-2} \int_{(0)}(2 \pi)^{2 \xi}\left\{\cos \left(\frac{1}{2} \pi(u-v)\right)-\sin \left(\pi\left(\frac{1}{2}(z-w)+\xi\right)\right)\right\} \\
& \quad \times S(\xi ; u, v, w, z) \Gamma(1-2 \xi)\{\zeta(2 \xi) \zeta(1+2 \xi)\}^{-1} \Xi(\xi ; u, v, w, z ; g) \mathrm{d} \xi .
\end{aligned}
$$

We then choose $Q$ which is to satisfy the condition

$$
3 B<Q \leqslant \frac{1}{4} A ; \quad \zeta(s) \neq 0 \quad \text { for } \operatorname{Im} s= \pm Q .
$$

We divide the range of integration in (3.48) into two parts according as $|\xi|>$ $Q$ and $|\xi| \leqslant Q$, and denote the corresponding parts of $J_{3, c}$ by $J_{3, c}^{(1)}$ and $f_{3, c}^{(2)}$, respectively. We observe that if $\operatorname{Re} \xi=0,|\operatorname{Im} \xi| \geqslant Q$, then $S(\xi ; u, v, w, z)$ is regular and $O\left(|\xi|^{c B}\right)$ uniformly in $\mathcal{B}$ with an absolute constant $c$. Then, Lemma 7 implies that the integrand in the part $J_{3, c}^{(1)}$ is regular and of fast decay with respect to $\xi$ uniformly in $\mathcal{B}$. Hence $\mathfrak{J}_{3, c}^{(1)}$ is regular in $\mathcal{B}$. As to $\mathfrak{f}_{3, c}^{(2)}$, we move the path to $L_{Q}$ which is the result of connecting the points $-Q i,[Q]+\frac{1}{4}-Q i,[Q]+\frac{1}{4}+Q i$, $Q i$ with straight lines. The singularities of the integrand which we encounter in this procedure are all poles, and located at

$$
\begin{aligned}
\frac{1}{2}(u+v+w+z-3), & \frac{1}{2}(u+z-v-w-1), \quad \frac{1}{2}(v+z-u-w-1) ; \\
\frac{1}{2} \rho & (|\operatorname{Im} \rho|<Q) ; \quad \frac{1}{2} n \quad(2 \leqslant n \leqslant[Q]) ;
\end{aligned}
$$

where $\rho$ is a complex zero of $\zeta(s)$; note our choice of $Q$. The first three come from $S(\xi ; u, v, w, z)$, and the others from $\Gamma(1-2 \xi) \zeta(2 \xi)^{-1}$, since we have here $(u, v, w, z) \in \mathcal{E}$ and so the $\Xi$-factor is regular for $\operatorname{Re} \xi \geqslant 0$. We may suppose, for an obvious reason, that the poles given in (3.50) are all simple, and do not coincide with any of those given in (3.51). Then we have

$$
\mathcal{J}_{3, c}(u, v, w, z ; g)=F_{-}(u, v, w, z ; g)+U(u, v, w, z ; g)+\mathcal{J}_{3, c}^{(Q)}(u, v, w, z ; g) .
$$

Here $F_{-}$and $U$ are the contributions of residues at the poles given in (3.50) and (3.51), respectively; and $J_{3, c}^{(Q)}$ is the same as (3.48) but with the path $L_{Q}^{*}$ which is the sum of the path $L_{Q}$ and the half lines $(-i \infty,-Q i],[Q i, i \infty)$. By virtue of Lemma 7, the terms $F_{-}$and $U$ are meromorphic over $\mathcal{B}$, and $\mathfrak{g}_{3, c}^{(Q)}$ is regular there. 
Summing up, we have

Lemma 8. The function $\mathfrak{J}_{1}(u, v, w, z ; g)$ (see (3.11)) continues meromorphically to the domain $\mathcal{B}$. Thus the decomposition (3.9) holds throughout $\mathcal{B}$.

It remains for us only to specialize (3.9) by setting $(u, v, w, z)=\mathrm{p}_{\tau}$. This amounts to studying the local behaviour, near $\mathrm{p}_{\tau}$, of the various components of $\partial_{1}$ which have been introduced in the above discussion. As a consequence we shall obtain the explicit formula for $\mathcal{L}_{4}(g ; \tau, \tau)$ furnished by Theorem 1 . Namely we have the decomposition, over $\mathcal{B}$,

$$
\mathcal{J}_{1}(u, v, w, z ; g)=\left\{\mathcal{J}_{2}+\mathcal{J}_{3, c}+\mathcal{J}_{3, d}^{+}+\mathcal{J}_{3, d}^{-}+\mathcal{J}_{3, h}^{+}\right\}(u, v, w, z ; g),
$$

where $\mathcal{J}_{3, d}^{ \pm}$is regular at $\mathrm{p}_{\tau}$. To see this we observe that when $(u, v, w, z)$ is near $\mathrm{p}_{\tau}$ the point $(i r, u, v, w, z)$, with an arbitrary real $r$, is not in the set $\mathcal{N}$ defined at (3.42); thus by $(3.25)-(3.26)$ the functions $\Phi_{ \pm}(i r ; u, v, w, z)$ are also regular at $\mathrm{p}_{\tau}$ for any real $r$. Hence $\mathcal{J}_{3, d}^{ \pm}$are regular at $\mathrm{p}_{\tau}$. Similarly one can see that $\mathcal{J}_{3, h}^{+}$ is regular at $\mathrm{p}_{\tau}$. That is, we may set $(u, v, w, z)=\mathrm{p}_{\tau}$ in the series expansions (3.36), (3.37) and (3.40) without any modification, and find that

$$
\begin{aligned}
& \left\{\mathcal{J}_{3, d}^{+}+\mathcal{J}_{3, d}^{-}+\mathcal{J}_{3, h}^{+}\right\}\left(\mathrm{p}_{\tau} ; g\right) \\
& =\sum_{j=1}^{\infty} \alpha_{j} H_{j}^{2}\left(\frac{1}{2}\right) H_{j}\left(2 \tau-\frac{1}{2}\right)\left\{\Phi_{+}+\Phi_{-}\right\}\left(i \kappa_{j} ; \mathrm{p}_{\tau} ; g\right) \\
& \quad+\sum_{k=1}^{\infty} \sum_{j=1}^{\vartheta(2 k)} \alpha_{j, 2 k} H_{j, 2 k}^{2}\left(\frac{1}{2}\right) H_{j, 2 k}\left(2 \tau-\frac{1}{2}\right) \Xi\left(2 k-\frac{1}{2} ; \mathrm{p}_{\tau} ; g\right),
\end{aligned}
$$

where we have used the fact that $H_{j}\left(\frac{1}{2}\right)=0$ if $\epsilon_{j}=-1$, and $H_{j, k}\left(\frac{1}{2}\right)=0$ if $k$ is odd. Note also that by (3.25)-(3.26) we have, for real $r$,

$$
\begin{aligned}
\left\{\Phi_{+}+\Phi_{-}\right\}\left(i r ; \mathrm{p}_{\tau} ; g\right)= & \frac{1}{4}\left(1+\frac{i}{\sinh (\pi r)}\right) \Xi\left(i r ; \mathrm{p}_{\tau} ; g\right) \\
& +\frac{1}{4}\left(1-\frac{i}{\sinh (\pi r)}\right) \Xi\left(-i r ; \mathrm{p}_{\tau} ; g\right) .
\end{aligned}
$$

On the other hand, Lemma 7 gives

$$
\begin{aligned}
\Xi\left(i r ; \mathrm{p}_{\tau} ; g\right)= & \frac{\Gamma\left(\frac{1}{2}+i r\right)^{2}}{\Gamma(1+2 i r)} \int_{0}^{\infty} y^{2 \tau-\frac{3}{2}+i r}(1+y)^{-\tau} g^{*}(\log (1+y)) \\
& \times F\left(\frac{1}{2}+i r, \frac{1}{2}+i r ; 1+2 i r ;-y\right) \mathrm{d} y
\end{aligned}
$$

Hence

$$
\begin{aligned}
& \left\{\Phi_{+}+\Phi_{-}\right\}\left(i r ; \mathrm{p}_{\tau} ; g\right)=\frac{1}{2} \int_{0}^{\infty} y^{2 \tau-\frac{3}{2}}(1+y)^{-\tau} g^{*}(\log (1+y)) \\
& \quad \times \operatorname{Re}\left\{y^{i r}\left(1+\frac{i}{\sinh (\pi r)}\right) \frac{\Gamma\left(\frac{1}{2}+i r\right)^{2}}{\Gamma(1+2 i r)} F\left(\frac{1}{2}+i r, \frac{1}{2}+i r ; 1+2 i r ;-y\right)\right\} \mathrm{d} y
\end{aligned}
$$


Further, we observe that (3.56) holds with ir replaced by $k-\frac{1}{2}$; and thus (3.57) gives, for any integer $k \geqslant 1$,

$$
\left\{\Phi_{+}+\Phi_{-}\right\}\left(2 k-\frac{1}{2} ; \mathrm{p}_{\tau} ; g\right)=\Xi\left(2 k-\frac{1}{2} ; \mathrm{p}_{\tau} ; g\right) .
$$

Now, we consider $\mathcal{J}_{3, c}$ in an immediate neighbourhood of $\mathrm{p}_{\tau}$. Let us assume first that

$$
\frac{1}{2}<\tau<\frac{3}{4}
$$

This is much similar to the case $\tau=\frac{1}{2}$, which is treated in [Mo6]. We return to (3.52), and move the contour in $g_{3, c}^{(Q)}$ back to the imaginary axis, while keeping $(u, v, w, z)$ close to $\mathrm{p}_{\tau}$. The poles which we encounter in this process are those given in (3.51) and $\frac{1}{2}(3-u-v-w-z)$, which is in fact to the right of the imaginary axis. Other poles of $S(\xi ; u, v, w, z)$ are either on the left of the imaginary axis or cancelled by the zeros of the factor $\cos \left(\frac{1}{2} \pi(u-v)\right)-\sin \left(\pi\left(\frac{1}{2}(z-w)+\xi\right)\right)$, and moreover Lemma 7 implies that $\Xi(\xi ; u, v, w, z ; g)$ is regular for $\operatorname{Re}(\xi) \geqslant-\frac{1}{4}$. We denote by $F_{+}(u, v, w, z ; g)$ the contribution of the pole $\frac{1}{2}(3-u-v-w-z)$. Then we have

$$
\mathcal{J}_{3, c}^{(Q)}(u, v, w, z ; g)=F_{+}(u, v, w, z ; g)-U(u, v, w, z ; g)+\mathcal{J}_{3, c}^{*}(u, v, w, z ; g)
$$

where $\mathcal{J}_{3, c}^{*}$ has the same expression as the right side of (3.48) but with different $(u, v, w, z)$. Hence, by $(3.52)$,

$$
\mathcal{J}_{3, c}(u, v, w, z ; g)=\left\{F_{+}+F_{-}\right\}(u, v, w, z ; g)+\mathcal{J}_{3, c}^{*}(u, v, w, z ; g) \text {, }
$$

when $(u, v, w, z)$ is close to $\mathrm{p}_{\tau}$. Here we should note that $\mathcal{J}_{3, c}^{*}$ is regular at $\mathrm{p}_{\tau}$, and

$$
\partial_{3, c}^{*}\left(\mathrm{p}_{\tau} ; g\right)=\frac{1}{\pi} \int_{-\infty}^{\infty} \frac{\left|\zeta\left(\frac{1}{2}+i t\right)\right|^{4}\left|\zeta\left(2 \tau-\frac{1}{2}+i t\right)\right|^{2}}{|\zeta(1+2 i t)|^{2}}\left\{\Phi_{+}+\Phi_{-}\right\}\left(i t ; \mathrm{p}_{\tau} ; g\right) \mathrm{d} t .
$$

This ends the local study of the decomposition (3.53) in the vicinity of $\mathrm{p}_{\tau}$, provided that (3.59) holds.

Now, if $(u, v, w, z)$ is close to $\mathrm{p}_{\tau}$, then we have

$$
\begin{aligned}
& \mathcal{J}(u, v, w, z ; g)=M(u, v, w, z ; g)+\mathcal{J}_{3, c}^{*}(u, v, w, z ; g)+\overline{\mathcal{J}_{3, c}^{*}(\bar{w}, \bar{z}, \bar{u}, \bar{v} ; g)} \\
& \quad+\left\{\mathcal{J}_{3, d}^{-}+\mathcal{J}_{3, d}^{+}+\mathcal{J}_{3, h}^{+}\right\}(u, v, w, z ; g)+\overline{\left\{\mathcal{J}_{3, d}^{-}+\mathcal{J}_{3, d}^{+}+\mathcal{J}_{3, h}^{+}\right\}(\bar{w}, \bar{z}, \bar{u}, \bar{v} ; g)}
\end{aligned}
$$

where

$$
\begin{aligned}
& M(u, v, w, z ; g)=\partial_{0}(u, v, w, z ; g)+\partial_{2}(u, v, w, z ; g)+\overline{\partial_{2}(\bar{w}, \bar{z}, \bar{u}, \bar{v} ; g)} \\
& \quad+\left\{F_{+}+F_{-}\right\}(u, v, w, z ; g)+\overline{\left\{F_{+}+F_{-}\right\}(\bar{w}, \bar{z}, \bar{u}, \bar{v} ; g)} .
\end{aligned}
$$


It should be stressed that all terms in (3.63) are regular at $\mathrm{p}_{\tau}$. That the function $M$ is regular at $\mathrm{p}_{\tau}$ is due to the fact that all terms in (3.63) except for $M$ have already been proved to be regular at $\mathrm{p}_{\tau}$.

It remains for us to express $M\left(\mathrm{p}_{\tau} ; g\right)$ in terms of $g$. We have

$$
M(u, v, w, z ; g)=\sum_{j=0}^{12} M_{j}(u, v, w, z ; g)
$$

with

$$
M_{6+j}(u, v, w, z ; g)=\overline{M_{j}(\bar{w}, \bar{z}, \bar{u}, \bar{v} ; g)} \quad(1 \leqslant j \leqslant 6) .
$$

Here (recall that $\tilde{g}$ is given by (3.1))

$$
\begin{aligned}
& M_{0}(u, v, w, z ; g)= g^{*}(0) \zeta(u+w) \zeta(u+z) \zeta(v+w) \\
& \times \zeta(v+z)\{\zeta(u+v+w+z)\}^{-1} \\
& M_{1}(u, v, w, z ; g)= \tilde{g}(v+w-1, w) \zeta(u+z) \zeta(v+w-1) \\
& \times \zeta(z-w+1) \zeta(u-v+1)\{\zeta(u+z-v-w+2)\}^{-1}, \\
& M_{2}(u, v, w, z ; g)= \tilde{g}(u+w-1, w) \zeta(v+z) \zeta(u+w-1) \\
& \times \zeta(z-w+1) \zeta(v-u+1)\{\zeta(v+z-u-w+2)\}^{-1}, \\
& M_{3}(u, v, w, z ; g)=(2 \pi)^{w-z}\left\{\cos \left(\frac{1}{2} \pi(u-v)\right)+\cos \left(\pi\left(z-w+\frac{1}{2}(u-v)\right)\right)\right\} \\
& \times \zeta(u+z-1) \zeta(v+w) \zeta(z-w) \zeta(v-u+1) \\
& \times\left\{\cos \left(\frac{1}{2} \pi(u+z-v-w)\right) \zeta(2-u-z+v+w)\right\}^{-1} \\
& \times \Xi\left(\frac{1}{2}(u+z-v-w-1) ; u, v, w, z ; g\right), \\
&(2 \pi)^{w-z}\left\{\cos \left(\frac{1}{2} \pi(u-v)\right)+\cos \left(\pi\left(z-w+\frac{1}{2}(v-u)\right)\right)\right\} \\
& \times\left\{\left(\cos \left(\frac{1}{2} \pi(v+z-u-w)\right) \zeta(2-v-z+u+w)\right\}^{-1}\right. \\
& \times \Xi\left(\frac{1}{2}(v+z-u-w-1) ; u, v, w, z ; g\right), \\
& M_{4}(u, v, w, z ; g)-(2 \pi)^{w-z}\left\{\cos \left(\frac{1}{2} \pi(u-v)\right)-\cos \left(\pi\left(z+\frac{1}{2}(u+v)\right)\right)\right\} \\
& \times \zeta(u+z-1) \zeta(2-v-w) \zeta(v+z-1) \zeta(2-u-w) \\
& \times\left\{\cos \left(\frac{1}{2} \pi(u+v+w+z)\right) \zeta(4-u-v-w-z)\right\}^{-1} \\
& \times \Xi\left(\frac{1}{2}(u+v+w+z-3) ; u, v, w, z ; g\right), \\
& M_{5}(u, v, w, z ; g)(2 \pi)^{w-z}\left\{\cos \left(\frac{1}{2} \pi(u-v)\right)+\cos \left(\pi\left(w+\frac{1}{2}(u+v)\right)\right)\right\} \\
& \times \zeta(u+z-1) \zeta(2-v-w) \zeta(v+z-1) \zeta(2-u-w) \\
& \times\left\{\cos \left(\frac{1}{2} \pi(u+v+w+z)\right) \zeta(4-u-v-w-z)\right\}^{-1} \\
& \times \Xi\left(-\frac{1}{2}(u+v+w+z-3) ; u, v, w, z ; g\right) . \\
& M_{6}(u, v, w, z ; g)
\end{aligned}
$$

Among these, $M_{0}$ is equal to $\mathfrak{J}_{0} ; M_{1}$ and $M_{2}$ come from $\mathfrak{J}_{2}$; and $M_{j}(3 \leqslant$ $j \leqslant 6)$ are the contributions of residues of the integral in (3.48) at the poles 
$\xi=\frac{1}{2}(u+z-v-w-1), \frac{1}{2}(v+z-u-w-1), \frac{1}{2}(u+v+w+z-3), \frac{1}{2}(3-u-v-w-z)$, respectively. They can be singular at $\mathrm{p}_{\tau}$ individually, but the singular parts should cancel each other out if they are brought into $(3.64)$, for $M$ is regular at $\mathrm{p}_{\tau}$. More precisely, put $(u, v, w, z)=\mathrm{p}_{\tau}+\left(a_{1}, a_{2}, a_{3}, a_{4}\right) \delta$ with a small complex $\delta$, and expand each term into a Laurent series in $\delta$; then the sum of the constant terms is equal to $M\left(\mathrm{p}_{\tau}\right)$, regardless of the choice of the vector $\left(a_{1}, a_{2}, a_{3}, a_{4}\right)$. We choose it in such a way that it is real and no singularities of any of the $M_{j}(0 \leqslant j \leqslant 12)$ are encountered when $|\delta|$ tends to 0 . This is possible, for the exceptional $a_{1}, a_{2}, a_{3}, a_{4}$ satisfy a finite number of linear relations. Thus we shall assume hereafter that $\delta \neq 0$ is small and the vector $\left(a_{1}, a_{2}, a_{3}, a_{4}\right)$ is chosen accordingly; and we denote $\left(a_{1}, a_{2}, a_{3}, a_{4}\right) \delta$ either by $(\delta)$ or by $\left(\delta_{1}, \delta_{2}, \delta_{3}, \delta_{4}\right)$. Also we denote the constant term of $M_{j}$ by $M_{j}^{*}$.

First, we have trivially

$$
M_{0}^{*}=M_{0}\left(\mathrm{p}_{\tau} ; g\right)=\frac{\zeta^{4}(2 \tau)}{\zeta(4 \tau)} g^{*}(0) \text {. }
$$

Invoking (3.1), we have

$$
\begin{aligned}
M_{1}\left(\mathrm{p}_{\tau}+(\delta) ; g\right)= & \Gamma\left(2 \tau-1+\delta_{2}+\delta_{3}\right) \zeta\left(2 \tau+\delta_{1}+\delta_{4}\right) \zeta\left(2 \tau-1+\delta_{2}+\delta_{3}\right) \\
& \times \zeta\left(\delta_{4}-\delta_{3}+1\right) \zeta\left(\delta_{1}-\delta_{2}+1\right)\left\{\zeta\left(2+\delta_{1}-\delta_{2}-\delta_{3}+\delta_{4}\right)\right\}^{-1} \\
& \times \int_{-\infty}^{\infty} \frac{\Gamma\left(1-\tau-\delta_{2}-i t\right)}{\Gamma\left(\tau+\delta_{3}-i t\right)} g(t) \mathrm{d} t
\end{aligned}
$$

This implies that the singularity of $M_{1}$ at $\mathrm{p}_{\tau}$ is of order two. Hence the constant term of $M_{1}\left(\mathrm{p}_{\tau}+(\delta) ; g\right)$ is a linear combination of the first three coefficients of the power series in $\delta$ for the last integral. Thus

$$
\begin{aligned}
& M_{1}^{*}=\int_{-\infty}^{\infty} \frac{\Gamma(1-\tau-i t)}{\Gamma(\tau-i t)} \\
& \times\left(d_{0}+d_{1} \frac{\Gamma^{\prime}}{\Gamma}(1-\tau-i t) \frac{\Gamma^{\prime}}{\Gamma}(\tau-i t)+d_{2} \frac{\Gamma^{\prime \prime}}{\Gamma}(1-\tau-i t)+d_{3} \frac{\Gamma^{\prime \prime}}{\Gamma}(\tau-i t)\right) g(t) \mathrm{d} t
\end{aligned}
$$

where the constants $d_{j}$ depend on $\tau$ and the vector $\left(a_{1}, a_{2}, a_{3}, a_{4}\right)$. Clearly $M_{2}$ can be treated in just the same way, and $M_{2}^{*}$ has the same form as (3.76).

The terms $M_{j}(3 \leqslant j \leqslant 6)$ are not so simple; and our computation of them depends on a classical formula of Barnes (see e.g., [WW]). By the definition (3.24) we have, for the $\Xi$-factor in $M_{3}$,

$$
\begin{aligned}
& \Xi\left(\frac{1}{2}(u+z-v-w-1) ; u, v, w, z ; g\right) \\
& =\frac{1}{2 \pi i} \int_{-i \infty}^{i \infty} \Gamma(u+z-1-s) \Gamma(s+1-u-w) \tilde{g}(s, w) \mathrm{d} s
\end{aligned}
$$


where the path separates the poles of $\Gamma(u+z-1-s)$ and those of the other two factors to the right and the left, respectively; that we can draw such a path is assured by our choice of $\left(a_{1}, a_{2}, a_{3}, a_{4}\right)$. Inserting (3.1) in this we get an absolutely convergent double integral, hence it follows that

$$
\begin{aligned}
& \Xi\left(\frac{1}{2}(u+z-v-w-1) ; u, v, w, z ; g\right)=\int_{-\infty}^{\infty} \frac{g(t)}{\Gamma(w-i t)} \\
& \quad \times \frac{1}{2 \pi i} \int_{-i \infty}^{i \infty} \Gamma(s) \Gamma(s+1-u-w) \Gamma(u+z-1-s) \Gamma(w-i t-s) \mathrm{d} s \mathrm{~d} t .
\end{aligned}
$$

The path of the inner integral is the same as in (3.1); and obviously we may suppose that it separates the poles of the first two $\Gamma$-factors from those of the other two. Hence we have, again by the Barnes formula,

$$
\begin{aligned}
& \Xi\left(\frac{1}{2}(u+z-v-w-1) ; u, v, w, z ; g\right) \\
& =\Gamma(u+z-1) \Gamma(z-w) \int_{-\infty}^{\infty} \frac{\Gamma(1-u-i t)}{\Gamma(z-i t)} g(t) \mathrm{d} t .
\end{aligned}
$$

This implies that $M_{3}$ has a singularity of order two at $\mathrm{p}_{\tau}$; thus $M_{3}^{*}$ admits an expression of the same form as (3.76). Obviously the same argument applies to $M_{4}$.

The $\Xi$-factor of $M_{5}$ can be computed in much the same way, and we have

$$
\begin{aligned}
& \Xi\left(\frac{1}{2}(u+v+w+z-3) ; u, v, w, z ; g\right) \\
& =\Gamma(u+z-1) \Gamma(v+z-1) \int_{-\infty}^{\infty} \frac{\Gamma(1-u-i t) \Gamma(1-v-i t)}{\Gamma(w-i t) \Gamma(z-i t)} g(t) \mathrm{d} t .
\end{aligned}
$$

This implies that $M_{5}$ is regular at $\mathrm{p}_{\tau}$, and

$$
\begin{aligned}
M_{5}^{*}=M_{5}\left(\mathrm{p}_{\tau} ; g\right)= & (1-\sec (2 \tau \pi)) \frac{(\Gamma(2 \tau-1) \zeta(2 \tau-1) \zeta(2-2 \tau))^{2}}{\zeta(4-4 \tau)} \\
& \times \int_{-\infty}^{\infty}\left(\frac{\Gamma(1-\tau-i t)}{\Gamma(\tau-i t)}\right)^{2} g(t) \mathrm{d} t
\end{aligned}
$$

A rearrangement gives

$$
\begin{aligned}
& \int_{-\infty}^{\infty}\left(\frac{\Gamma(1-\tau-i t)}{\Gamma(\tau-i t)}\right)^{2} g(t) \mathrm{d} t \\
& =\frac{1}{2 \pi^{2}} \int_{-\infty}^{\infty}|\Gamma(1-\tau+i t)|^{4}(1-\cos (2 \tau \pi) \cosh (2 \pi t)) g(t) \mathrm{d} t
\end{aligned}
$$

As to $M_{6}$, this also is regular at $\mathrm{p}_{\tau}$, since the $\Xi$-factor is regular there because of Lemma 7 . We have

$$
M_{6}^{*}=M_{6}\left(\mathrm{p}_{\tau} ; g\right)=(1+\sec (2 \tau \pi)) \frac{(\zeta(2 \tau-1) \zeta(2-2 \tau))^{2}}{\zeta(4-4 \tau)} \Xi\left(\frac{3}{2}-2 \tau ; \mathrm{p}_{\tau} ; g\right)
$$


with

$$
\begin{aligned}
& \Xi\left(\frac{3}{2}-2 \tau ; \mathrm{p}_{\tau} ; g\right) \\
& \quad=\frac{1}{2 i} \int_{-\infty}^{\infty} \frac{g(t)}{\Gamma(\tau-i t)}\left(\int_{\left(\sigma_{0}\right)} \frac{\Gamma(s+1-2 \tau)^{2} \Gamma(\tau-i t-s)}{\sin (\pi s) \Gamma(3-4 \tau+s)} \mathrm{d} s\right) \mathrm{d} t,
\end{aligned}
$$

where $2 \tau-1<\sigma_{0}<\tau$. We have

$$
\begin{aligned}
\int_{\left(\sigma_{0}\right)} \cdots= & -\frac{2 \pi i}{\sin (\pi(\tau-i t))} \sum_{j=1}^{N} \frac{\Gamma(j+1-2 \tau)^{2}}{\Gamma(j+3-4 \tau) \Gamma(j+1-\tau+i t)} \\
& +\frac{2 \pi i}{\sin (\pi(\tau-i t))} \sum_{j=0}^{N-1} \frac{\Gamma(j+1-\tau-i t)^{2}}{\Gamma(j+3(1-\tau)-i t) \Gamma(j+1)}+\int_{\left(\sigma_{N}\right)} \cdots
\end{aligned}
$$

where $2 \tau-1+N<\sigma_{N}<\tau+N$. This ends the discussion under the assumption that (3.59) holds.

Next, let

$$
\tau=\frac{3}{4}
$$

There is an essential difference between this case and (3.59), which we just discussed. This is due to the fact that the singularities $\pm \frac{1}{2}(u+v+w+z-3)$ of the integrand in (3.47) approach the origin as $(u, v, w, z)$ tends to $\mathrm{p}_{\frac{3}{4}}$. That is, they cannot be treated as well-separated.

While keeping $(u, v, w, z)$ close to $\mathrm{p}_{\frac{3}{4}}$, we move the contour in $\partial_{3, c}^{(Q)}$ to the imaginary axis but with a small outward indent around the origin. The poles which we encounter in this process are those given in (3.51) and $\pm \frac{1}{2}(u+v+w+z-3)$. Other poles of $S(\xi ; u, v, w, z)$ are either close to $-\frac{1}{2}$ or cancelled by the zeros of the factor $\cos \left(\frac{1}{2} \pi(u-v)\right)-\sin \left(\pi\left(\frac{1}{2}(z-w)+\xi\right)\right)$, and moreover Lemma 7 implies that $\Xi(\xi ; u, v, w, z ; g)$ is regular for $\operatorname{Re}(\xi) \geqslant-\frac{1}{4}$. We have

$$
\begin{aligned}
& \partial_{3, c}^{(Q)}(u, v, w, z ; g) \\
& =\left(F_{+}-F_{-}\right)(u, v, w, z ; g)-U(u, v, w, z ; g)+\partial_{3, c}^{*}(u, v, w, z ; g),
\end{aligned}
$$

where $F_{ \pm}$are as before, and $\mathcal{J}_{3, c}^{*}$ has the same expression as the right side of (3.47) but with the indented contour and a different $(u, v, w, z)$. By (3.52),

$$
\partial_{3, c}(u, v, w, z ; g)=F_{+}(u, v, w, z ; g)+\partial_{3, c}^{*}(u, v, w, z ; g) \text {, }
$$

when $(u, v, w, z)$ is close to $\mathrm{p}_{\frac{3}{4}}$. Here we should note that $\mathcal{J}_{3, c}^{*}$ is regular at $\mathrm{p}_{\frac{3}{4}}$, and

$$
\mathcal{J}_{3, c}^{*}\left(\mathrm{p}_{\frac{3}{4}} ; g\right)=\frac{1}{\pi} \int_{-\infty}^{\infty} \frac{\left|\zeta\left(\frac{1}{2}+i t\right)\right|^{4}|\zeta(1+i t)|^{2}}{|\zeta(1+2 i t)|^{2}}\left\{\Phi_{+}+\Phi_{-}\right\}\left(i t ; \mathrm{p}_{\frac{3}{4}} ; g\right) \mathrm{d} t,
$$


because this integrand is continuous. This ends the local study of the decomposition (3.53) in a small neighbourhood of $\mathrm{p}_{\frac{3}{4}}$.

Now, if $(u, v, w, z)$ is close to $\mathrm{p}_{\frac{3}{4}}$, then the counterpart of $(3.63)-(3.64)$ holds, and it remains for us to express $M\left(\mathrm{p}_{\frac{3}{4}} ; g\right)$ in terms of $g$, but with the new $M$. We have

$$
M(u, v, w, z ; g)=\sum_{\substack{j=0 \\ j \neq 5,11}}^{12} M_{j}(u, v, w, z ; g)
$$

where $M_{j}$ are the same as in (3.65)-(3.73). The terms $M_{5}$ and $M_{11}$ are missing, because the shift of the contour cancels the contribution of the pole at $\frac{1}{2}(u+v+$ $w+z-3)$ out, as we have seen above.

The computation of $M_{j}^{*}$ is the same as before. It should perhaps be remarked that

$$
M_{6}^{*}=M_{6}\left(\mathrm{p}_{\frac{3}{4}} ; g\right)=-\frac{2}{\pi} \zeta^{4}\left(\frac{1}{2}\right) \Xi\left(0 ; \mathrm{p}_{\frac{3}{4}} ; g\right) .
$$

We have

$$
\begin{aligned}
& \Xi\left(0 ; \mathrm{p}_{\frac{3}{4}} ; g\right) \\
& =\frac{1}{2 \pi i} \int_{-\infty}^{\infty} \frac{g(t)}{\Gamma\left(\frac{3}{4}-i t\right)}\left(\int_{\left(\frac{2}{3}\right)} \Gamma^{2}\left(s-\frac{1}{2}\right) \Gamma\left(\frac{3}{4}-i t-s\right) \Gamma(1-s) \mathrm{d} s\right) \mathrm{d} t \\
& =\pi \int_{-\infty}^{\infty}\left(\frac{\Gamma\left(\frac{1}{4}-i t\right)}{\Gamma\left(\frac{3}{4}-i t\right)}\right)^{2} g(t) \mathrm{d} t .
\end{aligned}
$$

Finally, let

$$
\frac{3}{4}<\tau<1
$$

Then the pole $\frac{1}{2}(3-u-v-w-z)$ is on the left of the imaginary axis; and the contribution of the pole $\frac{1}{2}(u+v+w+z-3)$ is cancelled out by moving the contour to the imaginary axis. That is, we have

$$
M(u, v, w, z ; g)=\sum_{\substack{j=0 \\ j \neq 5,6,11,12}}^{12} M_{j}(u, v, w, z ; g)
$$

This ends our discussion and completes the proof of Theorem 1.

\section{Sums of spectral values}

Note that (2.6) of Theorem 1 contains the quantities $\alpha_{j} H_{j}^{2}\left(\frac{1}{2}\right) H_{j}(\sigma)\left(\sigma=2 \tau-\frac{1}{2}\right)$ with a given $\frac{1}{2}<\sigma<1$, while in Part II of this work we shall encounter sums 
containing $\alpha_{j} H_{j}\left(\frac{1}{2}\right) H_{j}^{2}(\sigma)$. For the omega-results relating to moments we shall need the non-vanishing of

$$
L_{\sigma}(\kappa):=\sum_{\kappa_{j}=\kappa} \alpha_{j} H_{j}^{2}\left(\frac{1}{2}\right) H_{j}(\sigma), \quad N_{\sigma}(\kappa):=\sum_{\kappa_{j}=\kappa} \alpha_{j} H_{j}\left(\frac{1}{2}\right) H_{j}^{2}(\sigma)
$$

for infinitely many $\kappa$ and a given $\frac{1}{2}<\sigma<1$. The non-vanishing of $L_{\frac{1}{2}}(\kappa)$ was used (see [I2], [I5], [I7], [IM1], [Mo4], [Mo6]) for omega results on the fourth moment of $\left|\zeta\left(\frac{1}{2}+i t\right)\right|$. The non-vanishing of $L_{\sigma}(\kappa)$ and $N_{\sigma}(\kappa)$ that we need is a corollary of the following

Theorem 2. For fixed $\tau$ such that $\frac{1}{2}<\tau<1$ and $K \rightarrow \infty$, we have

$$
\sum_{\kappa_{j} \leqslant K} \alpha_{j} H_{j}\left(\frac{1}{2}\right) H_{j}^{2}(\tau)=(1+o(1)) \pi^{-2} \zeta^{2}\left(\tau+\frac{1}{2}\right) \zeta(2 \tau) K^{2}
$$

and

$$
\sum_{\kappa_{j} \leqslant K} \alpha_{j} H_{j}^{2}\left(\frac{1}{2}\right) H_{j}(\tau)=(1+o(1)) 2 \pi^{-2} \zeta^{2}\left(\tau+\frac{1}{2}\right) K^{2} \log K .
$$

For $L_{\frac{1}{2}}(\kappa)$ not only that non-vanishing is known, but a sharper asymptotic formula for the sum in question, namely

$$
\sum_{\kappa_{j} \leqslant K} \alpha_{j} H_{j}^{3}\left(\frac{1}{2}\right)=K^{2} P_{3}(\log K)+O\left(K^{5 / 4} \log ^{37 / 4} K\right),
$$

proved by the first author [Iv9], where $P_{3}(x)$ is a suitable cubic polynomial. One could also employ similar methods to obtain a sharpening of (4.2) and (4.3), but this will not be done here, since it is not needed in the sequel. It is known (see Katok-Sarnak $[\mathrm{KS}])$ that $H_{j}\left(\frac{1}{2}\right) \geqslant 0$; it follows trivially from the functional equation for $H_{j}(s)$ that $H_{j}\left(\frac{1}{2}\right)=0$ if $\varepsilon_{j}=-1$. Our formula (4.3) supports the conjecture that $H_{j}(\sigma) \geqslant 0$ for $0 \leqslant \sigma \leqslant 1$, but this remains an open problem.

Proof of Theorem 2. Because $H_{j}\left(\frac{1}{2}\right)=0$ when $\epsilon_{j}=-1$, we may start by treating the sum

$$
\sum_{\kappa_{j} \leqslant K}\left(\epsilon_{j} \alpha_{j} H_{j}\left(\frac{1}{2}\right) H_{j}(\lambda)\right) \cdot H_{j}(\tau)
$$

with the aim of taking later $\lambda=\frac{1}{2}$ or $\lambda=\tau$ in (4.5).

Let $h(r)$ be an even, entire function such that $h\left( \pm \frac{1}{2} i\right)=0$ and $h(r) \ll$ $\exp \left(-c|r|^{2}\right)(c>0)$ in any fixed horizontal strip, and

$$
H(u, v ; f ; h):=\sum_{j=1}^{\infty} \epsilon_{j} \alpha_{j} H_{j}(u) H_{j}(v) t_{j}(f) h\left(\kappa_{j}\right) \quad(f \geqslant 1) .
$$


Transformation formulas for the sums appearing in (4.6) were established by the second author [Mo1] and then in [Mo6] (see eq. (3.3.6) there, also (3.3.8) and (3.3.9) are important). The formulas in question transform the quantity (4.6) from spectral theory into a sum of various quantities from classical analytic number theory. We set

$$
\mathcal{H}(\lambda ; f ; h)=H\left(\frac{1}{2}, \lambda ; f ; h\right) .
$$

According to the formulas displayed on p. 117 of [Mo6], we have

$$
\begin{aligned}
& H(u, v ; f ; h)=2(\pi i)^{-1}(2 \pi \sqrt{f})^{2(u-1)} \frac{\hat{h}(1-u)}{\cos (\pi u)} \sigma_{1-u-v}(f) \zeta(1-u+v) \\
& +2(\pi i)^{-1}(2 \pi \sqrt{f})^{2(v-1)} \frac{\hat{h}(1-v)}{\cos (\pi v)} \sigma_{1-u-v}(f) \zeta(1-v+u) \\
& +8(2 \pi)^{u+v-4} \sum_{m=1}^{\infty} m^{u-1} \sigma_{v-u}(m) \sigma_{1-u-v}(m+f) \Psi_{+}(u, v ; m / f ; h) \\
& +8(2 \pi)^{u+v-4} \sum_{m=1}^{\infty} m^{u-1} \sigma_{v-u}(m) \sigma_{1-u-v}(m-f) \Psi_{-}(u, v ; m / f ; h) \\
& +8(2 \pi)^{u+v-4} f^{u-1} \sigma_{v-u}(f) \zeta(u+v-1) \Psi_{-}(u, v ; 1 ; h) \\
& -4 \sigma_{2(u-1)}(f) f^{1-u} \zeta(u+v-1) \zeta(v-u+1) h(i(u-1)) / \zeta(3-2 u) \\
& -4 \sigma_{2(v-1)}(f) f^{1-v} \zeta(u+v-1) \zeta(u-v+1) h(i(v-1)) / \zeta(3-2 v) \\
& -\pi^{-1} \int_{-\infty}^{\infty} \frac{\sigma_{2 i r}(f) \zeta(u+i r) \zeta(u-i r) \zeta(v+i r) \zeta(v-i r)}{f^{i r}|\zeta(1+2 i r)|^{2}} h(r) \mathrm{d} r .
\end{aligned}
$$

Here

$$
\begin{aligned}
\Psi_{+}(u, v ; x ; h)= & -\int_{(\beta)} \Gamma(1-u-s) \Gamma(1-v-s) \\
& \times \cos \left(\pi\left(s+\frac{1}{2}(u+v)\right)\right) \frac{\hat{h}(s)}{\cos (\pi s)} x^{s} \mathrm{~d} s
\end{aligned}
$$

and

$$
\begin{aligned}
\Psi_{-}(u, v ; x ; h)= & \cos \left(\frac{1}{2} \pi(u-v)\right) \\
& \times \int_{(\beta)} \Gamma(1-u-s) \Gamma(1-v-s) \frac{\hat{h}(s)}{\cos (\pi s)} x^{s} \mathrm{~d} s,
\end{aligned}
$$

where

$$
\hat{h}(s)=\int_{\operatorname{Im} r=-C} r h(r) \frac{\Gamma(s+i r)}{\Gamma(1-s+i r)} \mathrm{d} r \quad(\operatorname{Re} s>-C),
$$

with any large $C>0$. This is proved if $u \neq v$, which can be dropped by an obvious convention; and $1+\beta<\operatorname{Re}(u), \operatorname{Re}(v)<1$ with $-\frac{3}{2}<\beta<0$. 
If

$$
\frac{1}{2}<\lambda<1,
$$

then we have

$$
\begin{aligned}
& \lim _{(u, v) \rightarrow\left(\frac{1}{2}, \lambda\right)}\left\{(2 \pi \sqrt{f})^{2(u-1)} \frac{\hat{h}(1-u)}{\cos (\pi u)} \sigma_{1-u-v}(f) \zeta(1-u+v)\right. \\
& \left.+(2 \pi \sqrt{f})^{2(v-1)} \frac{\hat{h}(1-v)}{\cos (\pi v)} \sigma_{1-u-v}(f) \zeta(1-v+u)\right\} \\
& =\frac{1}{2 \pi^{2}} \zeta\left(\lambda+\frac{1}{2}\right)(\hat{h})^{\prime}\left(\frac{1}{2}\right) \sigma_{\frac{1}{2}-\lambda}(f) f^{-\frac{1}{2}} \\
& +(2 \pi)^{2(\lambda-1)} \sec (\pi \lambda) \zeta\left(\frac{3}{2}-\lambda\right) \hat{h}(1-\lambda) \sigma_{\frac{1}{2}-\lambda}(f) f^{\lambda-1},
\end{aligned}
$$

where the fact $\hat{h}\left(\frac{1}{2}\right)=0$ has been used (see (3.3.15) of [Mo6]), and that

$$
\begin{aligned}
& \lim _{(u, v) \rightarrow\left(\frac{1}{2}, \lambda\right)}\left\{\sigma_{2(u-1)}(f) f^{1-u} \zeta(u+v-1) \zeta(v-u+1) h(i(u-1)) / \zeta(3-2 u)\right. \\
& \left.\quad+\sigma_{2(v-1)}(f) f^{1-v} \zeta(u+v-1) \zeta(u-v+1) h(i(v-1)) / \zeta(3-2 v)\right\} \\
& =\frac{6}{\pi^{2}} \zeta\left(\lambda-\frac{1}{2}\right) \zeta\left(\lambda+\frac{1}{2}\right) h\left(-\frac{1}{2} i\right) \sigma_{-1}(f) f^{\frac{1}{2}} \\
& \quad+\frac{\zeta\left(\lambda-\frac{1}{2}\right) \zeta\left(\frac{3}{2}-\lambda\right)}{\zeta(3-2 \lambda)} h(i(\lambda-1)) \sigma_{2(\lambda-1)}(f) f^{1-\lambda} .
\end{aligned}
$$

Note that the right sides of both (4.13) and (4.14) have removable singularities at $\lambda=\frac{1}{2}$. It is found that if (4.12) holds, then

$$
\mathcal{H}(\lambda ; f ; h)=\sum_{\nu=1}^{7} \mathcal{H}_{\nu}(\lambda ; f ; h) .
$$

Here

$$
\begin{aligned}
\mathcal{H}_{1}(\lambda ; f ; h) & =\frac{2}{\pi i} \times \text { the right side of }(4.13), \\
\mathcal{H}_{2}(\lambda ; f ; h) & =8(2 \pi)^{\lambda-\frac{7}{2}} \sum_{m=1}^{\infty} m^{-\frac{1}{2}} \sigma_{\lambda-\frac{1}{2}}(m) \sigma_{\frac{1}{2}-\lambda}(m+f) \Psi^{+}(\lambda ; m / f ; h), \\
\mathcal{H}_{3}(\lambda ; f ; h) & =8(2 \pi)^{\lambda-\frac{7}{2}} \\
& \times \sum_{m=1}^{\infty}(m+f)^{-\frac{1}{2}} \sigma_{\lambda-\frac{1}{2}}(m+f) \sigma_{\frac{1}{2}-\lambda}(m) \Psi^{-}(\lambda ; 1+m / f ; h), \\
\mathcal{H}_{4}(\lambda ; f ; h) & =8(2 \pi)^{\lambda-\frac{7}{2}} \sum_{m=1}^{f-1} m^{-\frac{1}{2}} \sigma_{\lambda-\frac{1}{2}}(m) \sigma_{\frac{1}{2}-\lambda}(f-m) \Psi^{-}(\lambda ; m / f ; h), \\
\mathcal{H}_{5}(\lambda ; f ; h) & =8(2 \pi)^{\lambda-\frac{7}{2}} f^{-\frac{1}{2}} \sigma_{\frac{1}{2}-\lambda}(f) \zeta\left(\lambda-\frac{1}{2}\right) \Psi^{-}(\lambda ; 1 ; h), \\
\mathcal{H}_{6}(\lambda ; f ; h) & =-4 \times \text { the right side of }(4.14), \\
\mathcal{H}_{7}(\lambda ; f ; h) & =-\pi^{-1} \int_{-\infty}^{\infty} \frac{\left|\zeta\left(\frac{1}{2}+i r\right)\right|^{2}|\zeta(\lambda+i r)|^{2}}{|\zeta(1+2 i r)|^{2}} \sigma_{2 i r}(f) f^{-i r} h(r) \mathrm{d} r,
\end{aligned}
$$


where

$$
\begin{gathered}
\Psi^{+}(\lambda ; x ; h)=-\int_{(\beta)} \Gamma\left(\frac{1}{2}-s\right) \Gamma(1-\lambda-s) \frac{\cos \left(\pi\left(s+\frac{1}{2}\left(\lambda+\frac{1}{2}\right)\right)\right.}{\cos \pi s} \hat{h}(s) x^{s} \mathrm{~d} s, \\
\Psi^{-}(x ; h)=\cos \left(\frac{1}{2} \pi\left(\frac{1}{2}-\lambda\right)\right) \int_{(\beta)} \Gamma\left(\frac{1}{2}-s\right) \Gamma(1-\lambda-s) \frac{\hat{h}(s)}{\cos \pi s} x^{s} \mathrm{~d} s
\end{gathered}
$$

with $-\frac{3}{2}<\beta<1-\lambda$. If we consider the limit as $\lambda$ tends to $\frac{1}{2}$, then from (4.15)-(4.24) we obtain the assertion of Lemma 3.8 of [Mo6].

We have

$$
(\hat{h})^{\prime}\left(\frac{1}{2}\right)=2 \int_{-\infty}^{\infty} r h(r) \frac{\Gamma^{\prime}}{\Gamma}\left(\frac{1}{2}+i r\right) \mathrm{d} r
$$

and

$$
\hat{h}(1-\lambda)=\int_{-\infty}^{\infty} r h(r) \frac{\Gamma(1-\lambda+i r)}{\Gamma(\lambda+i r)} \mathrm{d} r .
$$

Also,

$$
\begin{aligned}
& \Psi^{+}(\lambda ; x ; h)=-\frac{1}{\pi i} \int_{-\infty}^{\infty} r h(r) \sinh \pi r \\
& \quad \times \int_{(\beta)} \Gamma\left(\frac{1}{2}-s\right) \Gamma(1-\lambda-s) \Gamma(s+i r) \Gamma(s-i r) \cos \left\{\pi\left(s+\frac{1}{2}\left(\lambda+\frac{1}{2}\right)\right)\right\} x^{s} \mathrm{~d} s \mathrm{~d} r,
\end{aligned}
$$

with $0<\beta<1-\lambda$. Evaluating the inner integral, we have, for any $x>0$,

$$
\begin{aligned}
& \Psi^{+}(\lambda ; x ; h)=-2 \pi i \int_{-\infty}^{\infty} \frac{r h(r)}{\cosh \pi r} \cos \pi\left(i r-\frac{1}{2}\left(\lambda+\frac{1}{2}\right)\right) \\
& \quad \times \frac{\Gamma\left(\frac{1}{2}+i r\right) \Gamma(1-\lambda+i r)}{\Gamma(1+2 i r)} F\left(\frac{1}{2}+i r, 1-\lambda+i r ; 1+2 i r ;-1 / x\right) x^{-i r} \mathrm{~d} r .
\end{aligned}
$$

Then, by Gauss' integral representation for $F$ (see e.g., [L] or [WW]),

$$
\begin{aligned}
& \Psi^{+}(\lambda ; x ; h)=-2 \pi i \int_{0}^{1}\{y(1-y)\}^{-\frac{1}{2}}\left(1+\frac{y}{x}\right)^{\lambda-1} \\
& \quad \times \int_{-\infty}^{\infty} r h(r) \frac{\cos \pi\left(i r-\frac{1}{2}\left(\lambda+\frac{1}{2}\right)\right)}{\cosh \pi r} \frac{\Gamma(1-\lambda+i r)}{\Gamma\left(\frac{1}{2}+i r\right)}\left(\frac{y(1-y)}{x+y}\right)^{i r} \mathrm{~d} r \mathrm{~d} y,
\end{aligned}
$$

which corresponds to (3.3.41) of [Mo6].

In what concerns $\Psi^{-}$, for $x>1$ we obtain in a similar fashion

$$
\begin{aligned}
& \Psi^{-}(\lambda ; x ; h)=2 \pi i \cos \left(\frac{1}{2} \pi\left(\frac{1}{2}-\lambda\right)\right) \int_{-\infty}^{\infty} \frac{r h(r)}{\cosh (\pi r)} \\
& \quad \times \frac{\Gamma\left(\frac{1}{2}+i r\right) \Gamma(1-\lambda+i r)}{\Gamma(1+2 i r)} F\left(\frac{1}{2}+i r, 1-\lambda+i r ; 1+2 i r ; 1 / x\right) x^{-i r} \mathrm{~d} r \\
& =2 i \cos \left(\frac{1}{2} \pi\left(\frac{1}{2}-\lambda\right)\right) \int_{0}^{1}\{y(1-y)\}^{-\frac{1}{2}}\left(1-\frac{y}{x}\right)^{\lambda-1} \\
& \quad \times \int_{-\infty}^{\infty} r h(r) \Gamma\left(\frac{1}{2}-i r\right) \Gamma(1-\lambda+i r)\left(\frac{y(1-y)}{x-y}\right)^{i r} \mathrm{~d} r \mathrm{~d} y,
\end{aligned}
$$


which corresponds to (3.3.43) of [Mo6]. Also,

$$
\begin{aligned}
& \Psi^{-}(\lambda ; 1 ; h)=\frac{2 \pi \cos \left(\frac{1}{2} \pi\left(\frac{1}{2}-\lambda\right)\right)}{\Gamma\left(\frac{3}{2}-\lambda\right)} \\
& \quad \times \int_{-\infty}^{\infty} r h(r) \tanh (\pi r) \Gamma(1-\lambda+i r) \Gamma(1-\lambda-i r) \mathrm{d} r .
\end{aligned}
$$

When $0<x<1$, we argue as on p. 121 of [Mo6], to deduce that

$$
\begin{aligned}
\Psi^{-}(x ; h)= & \cos \left(\frac{1}{2} \pi\left(\frac{1}{2}-\lambda\right)\right) \int_{0}^{\infty}\left\{\int_{-\infty}^{\infty} r h(r)\left(\frac{y}{1+y}\right)^{i r} \mathrm{~d} r\right\} \\
& \times\left\{\int_{(\beta)} x^{s}(y(y+1))^{s-1} \frac{\Gamma\left(\frac{1}{2}-s\right) \Gamma(1-\lambda-s)}{\Gamma(1-2 s) \cos (\pi s)} \mathrm{d} s\right\} \mathrm{d} y,
\end{aligned}
$$

with $-\frac{3}{2}<\beta<1-\lambda, \beta \neq-\frac{1}{2}$.

We shall now derive an approximate functional equation for $H_{j}(\tau)$. The expression (4.5) implies in particular that we may restrict ourselves to the case $\varepsilon_{j}=+1$. Let us assume that

$$
\left|\kappa_{j}-K\right| \leqslant G \log K, \quad(\log K)^{2}<G<K^{1-\delta} \quad(\delta>0) .
$$

Take a large $C>0$ and consider the integral

$$
\mathcal{R}:=\frac{1}{2 \pi i \mu} \int_{(3)} H_{j}(w+\tau) K^{w} \Gamma(w / \mu) \mathrm{d} w \quad(\mu=C \log K) .
$$

We have

$$
\mathcal{R}=\sum_{f \leqslant 3 K} t_{j}(f) f^{-\tau} \exp \left(-(f / K)^{\mu}\right)+O\left(\mathrm{e}^{-K}\right) .
$$

Shifting the path of integration in (4.34) to $\operatorname{Re} w=-\frac{1}{2} \mu$ and recalling the functional equation for $H_{j}(s)$ (see (3.24) of [Mo6]), we obtain

$$
\mathcal{R}=H_{j}(\tau)+\sum_{f=1}^{\infty} t_{j}(f) f^{\tau-1} \mathcal{R}_{j}(f K)
$$

where

$$
\begin{aligned}
\mathcal{R}_{j}(x):= & \frac{1}{(2 \pi)^{2(1-\tau)} \pi i \mu} \\
& \times \int_{\left(-\frac{1}{2} \mu\right)}\left(4 \pi^{2} x\right)^{w} \Gamma\left(1-\tau-w+i \kappa_{j}\right) \Gamma\left(1-\tau-w-i \kappa_{j}\right) \\
& \times\left\{\cosh \left(\pi \kappa_{j}\right)-\cos (\pi(w+\tau))\right\} \Gamma(w / \mu) \mathrm{d} w .
\end{aligned}
$$


By Stirling's formula for the gamma-function the above integrand is

$$
\ll\left(4 \pi^{2} x\right)^{-\frac{1}{2} \mu}\left(\left|w+i \kappa_{j}\right|\left|w-i \kappa_{j}\right|\right)^{\frac{1}{2}(\mu+1)-\tau} \exp (-\pi|w| /(2 \mu))
$$

and thus

$$
\mathcal{R}_{j}(x)=O\left(K^{1-2 \tau}\left(4 \pi^{2} x K^{-2}\right)^{-\frac{1}{2} \mu}\right),
$$

where the implied constant is absolute. This allows us to truncate the last sum over $f$ at $f=[3 K]$ with an error which is $\ll K^{-C}$ for any fixed $C>0$. Hence we have proved

Lemma 10. For $\frac{1}{2}<\tau<1$ fixed and uniformly for all $\kappa_{j}$ satisfying (4.33) and $\varepsilon_{j}=1$,

$$
\begin{aligned}
H_{j}(\tau)= & \sum_{f \leqslant 3 K} t_{j}(f) f^{-\tau} \exp \left(-(f / K)^{\mu}\right) \\
& -\sum_{f \leqslant 3 K} t_{j}(f) f^{\tau-1} \mathcal{R}_{j}^{(1)}(f K)+O\left(K^{-\frac{1}{2} C}\right),
\end{aligned}
$$

where $C>0$ is any given constant and

$$
\begin{aligned}
\mathcal{R}_{j}^{(1)}(x):= & \frac{1}{(2 \pi)^{2(1-\tau)} \pi i \mu} \\
& \times \int_{-\mu^{-1}-i \mu^{2}}^{-\mu^{-1}+i \mu^{2}}\left(4 \pi^{2} x\right)^{w} \Gamma\left(1-\tau-w+i \kappa_{j}\right) \Gamma\left(1-\tau-w-i \kappa_{j}\right) \\
& \times\left\{\cosh \left(\pi \kappa_{j}\right)-\cos (\pi(w+\tau))\right\} \Gamma(w / \lambda) \mathrm{d} w
\end{aligned}
$$

Stirling's formula gives, for any $N \geqslant 1$ and for the values of $w$ relevant in (4.41),

$$
\begin{aligned}
& \log \Gamma\left(1-\tau-w+i \kappa_{j}\right)=\left(\frac{1}{2}-\tau-w+i \kappa_{j}\right) \log \left(1-\tau-w+i \kappa_{j}\right) \\
& \quad+\tau-1+w-i \kappa_{j}+\frac{1}{2} \log (2 \pi)+\sum_{\nu=1}^{2 N} b_{\nu}\left(1-\tau-w+i \kappa_{j}\right)^{-\nu}+O\left(K^{-2 N-\frac{1}{2}}\right)
\end{aligned}
$$

where $b_{\nu}$ 's are absolute constants, and the implied constant depends only on $N$. Therefore

$$
\begin{aligned}
& \log \Gamma\left(1-\tau-w+i \kappa_{j}\right)=\left(\frac{1}{2}-\tau-w+i \kappa_{j}\right)\left\{\log \left(\kappa_{j}\right)+\frac{1}{2} \pi i\right\} \\
& -i \kappa_{j}+\frac{1}{2} \log (2 \pi)+\sum_{\nu=1}^{2 N} p_{\nu}(w) \kappa_{j}^{-\nu}+O\left(K^{-2 N}(\log K)^{12 N+6}\right)
\end{aligned}
$$


with certain polynomials $p_{\nu}$ of degree $\leqslant \nu+1$ with constant coefficients. Adding to this the corresponding formula for $\Gamma\left(1-\tau-w-i \kappa_{j}\right)$, we have

$$
\begin{aligned}
& \log \left\{\Gamma\left(1-\tau-w+i \kappa_{j}\right) \Gamma\left(1-\tau-w-i \kappa_{j}\right)\right\} \\
& =(1-2 \tau-2 w) \log \left(\kappa_{j}\right)-\pi \kappa_{j} \\
& \quad+\log (2 \pi)+\sum_{\nu=1}^{N} p_{2 \nu}(w) \kappa_{j}^{-2 \nu}+O\left(K^{-2 N}(\log K)^{12 N+6}\right) .
\end{aligned}
$$

This implies readily that the integrand of (4.41) can be replaced by

$$
\pi \kappa_{j}^{1-2 \tau}\left(4 \pi^{2} \kappa_{j}^{-2} x\right)^{w}\left\{1+\sum_{\nu=1}^{N} q_{\nu}(w) \kappa_{j}^{-2 \nu}+O\left(K^{-N}\right)\right\} \Gamma(w / \lambda),
$$

where $q_{\nu}(w)$ are polynomials of degree $\leqslant 3 \nu$ with constant coefficients, and the $O$-constant depends only on $N$. Then we expand each $\kappa_{j}^{1-2 \tau-2 w-2 \nu}$ into a power series in $\left(1-\left(\kappa_{j} / K\right)^{2}\right)=O\left(K^{-\delta} \log K\right)$ and truncate it at the power $N_{1}=[2 N / \delta]$. Rearranging the result of truncation we see that the integrand of (4.41) can be written as

$$
\pi K^{1-2 \tau}\left(4 \pi^{2} K^{-2} x\right)^{w}\left\{Q\left(w, 1-\left(\kappa_{j} / K\right)^{2}\right)+O\left(K^{-N}\right)\right\} \Gamma(w / \lambda),
$$

where

$$
Q(w, y)=\sum_{\nu=0}^{N_{1}} u_{\nu}(w) y^{\nu}, u_{0}(w)=1+\sum_{\nu=1}^{N} q_{\nu}(w) K^{-2 \nu} .
$$

Inserting (4.47) into (4.41) and restoring the range of integration to the whole line $\operatorname{Re} w=-\mu^{-1}$, we get, uniformly for $f \leqslant 3 K$,

$$
\mathcal{R}_{j}^{(1)}(f K)=\left(\frac{K}{\pi}\right)^{1-2 \tau} \sum_{\nu=0}^{N_{1}} U_{\nu}(f K)\left(1-\left(\kappa_{j} / K\right)^{2}\right)^{\nu}+O\left(K^{-N}\right) .
$$

Here $N_{1}=[2 N / \delta]$ and

$$
U_{\nu}(x)=\frac{1}{2 \pi i \mu} \int_{\left(-\mu^{-1}\right)}\left(4 \pi^{2} K^{-2} x\right)^{w} u_{\nu}(w) \Gamma(w / \mu) \mathrm{d} w,
$$

where $u_{p}(w)$ is a polynomial of degree $\leqslant 2 N_{1}$, whose coefficients are independent of $\kappa_{j}$ and bounded by a constant depending only on $\delta, \tau$, and $N$. Hence, if $\varepsilon_{j}=1$, we have, for any $N \geqslant 1$ and $\mu=C \log K$ with a sufficiently large $C>0$,

$$
\begin{aligned}
& H_{j}(\tau)=\sum_{f \leqslant 3 K} t_{j}(f) f^{-\tau} \exp \left(-(f / K)^{\mu}\right) \\
& -\left(\frac{K}{\pi}\right)^{1-2 \tau} \sum_{\nu=0}^{N_{1}} \sum_{f \leqslant 3 K} t_{j}(f) f^{\tau-1} U_{\nu}(f K)\left(1-\left(\kappa_{j} / K\right)^{2}\right)^{\nu}+O\left(K^{-\frac{1}{5} N}+K^{-\frac{1}{2} C}\right)
\end{aligned}
$$

with the implied constant depending only on $\delta, \tau, C$, and $N$. 
We shall evaluate asymptotically, as $K \rightarrow \infty$,

$$
\mathcal{C}(\lambda, \tau ; K, G):=\sum_{j=1}^{\infty} \alpha_{j} H_{j}\left(\frac{1}{2}\right) H_{j}(\lambda) H_{j}(\tau) h_{0}\left(\kappa_{j}\right),
$$

where $\frac{1}{2}<\lambda, \tau<1$ initially, and the weight function will be

$$
h_{0}(r):=\left(r^{2}+\frac{1}{4}\right)\left\{\exp \left(-\left(\frac{r-K}{G}\right)^{2}\right)+\exp \left(-\left(\frac{r+K}{G}\right)^{2}\right)\right\},
$$

provided that (4.33) holds. From (4.50) we obtain

$$
\begin{aligned}
& \mathcal{C}(\lambda, \tau ; K, G)=\sum_{f \leqslant 3 K} f^{-\tau} \exp \left(-(f / K)^{\mu}\right) \mathcal{H}\left(\lambda ; f ; h_{0}\right) \\
& -\left(\frac{K}{\pi}\right)^{1-2 \tau} \sum_{\nu=0}^{N_{1}} \sum_{f \leqslant 3 K} f^{\tau-1} U_{\nu}(f K) \mathcal{H}\left(\lambda ; f ; h_{\nu}\right)+o(1),
\end{aligned}
$$

where $\mathcal{H}$ is defined by (4.7), and

$$
h_{\nu}(r)=h_{0}(r)\left(1-(r / K)^{2}\right)^{\nu} .
$$

To evaluate $\mathcal{H}\left(\lambda ; f ; h_{\nu}\right)$, we use (4.15). The contributions of (4.17), (4.18), (4.20) and (4.21) are negligible, which can be confirmed in much the same way as on pp. 128-129 of [Mo6]. Then, corresponding to (3.4.25) there, we have $(d(n) \equiv$ $\sigma_{0}(n)$ is the number of divisors of $n$ )

$$
\begin{aligned}
& \mathcal{H}\left(\lambda ; f ; h_{\nu}\right)=\mathcal{H}_{1}\left(\lambda ; f ; h_{\nu}\right)+O\left(d(f)\left(G+K^{\frac{2}{3}}\right) K^{2}(G / K)^{\nu}(\log K)^{c}\right), \\
& +O\left(f^{\frac{3}{2}} K^{3} G^{-3}(G / K)^{\nu} \log K \sum_{m<f} m^{-2} \sigma_{\lambda-\frac{1}{2}}(m) \sigma_{\frac{1}{2}-\lambda}(f-m)\right)
\end{aligned}
$$

with some constant $c>0$, provided that

$$
K^{\frac{1}{2}+\delta}<G<K^{1-\delta} .
$$

Note that (4.55) holds with $\lambda=\frac{1}{2}$, too, and that the estimation of the error terms is not the best that our argument can attain. Also, we should remark that

$$
\mathcal{H}_{1}\left(\lambda ; f ; h_{\nu}\right) \ll K^{3} G(G / K)^{\nu}(\log f K) \sigma_{\frac{1}{2}-\lambda}(f) f^{-\frac{1}{2}},
$$

uniformly for $\frac{1}{2} \leqslant \lambda<1$ and for all $f \geqslant 1$. In fact, when $\frac{1}{2}+(\log K)^{-1} \leqslant \lambda<1$, this follows from (4.16), (4.25), (4.26), and otherwise one may use the Taylor expansion at $\lambda=\frac{1}{2}$. 
Inserting (4.55) in (4.53) we obtain

$$
\begin{aligned}
\mathcal{C}(\lambda, \tau ; K, G) \\
=\sum_{f<3 K} \mathcal{H}_{1}\left(\lambda ; f ; h_{0}\right)\left(f^{-\tau} \exp \left(-(f / K)^{\mu}\right)-\left(\frac{K}{\pi}\right)^{1-2 \tau} f^{\tau-1} U_{0}(f K)\right) \\
\quad+O\left(K^{3-\tau} G(\log K)^{c}\right) \\
=\sum_{f=1}^{\infty} \mathcal{H}_{1}\left(\lambda ; f ; h_{0}\right)\left(f^{-\tau} \exp \left(-(f / K)^{\mu}\right)-\left(\frac{K}{\pi}\right)^{1-2 \tau} f^{\tau-1} U_{0}(f K)\right) \\
\quad+O\left(K^{3-\tau} G(\log K)^{c}\right),
\end{aligned}
$$

provided that $\frac{1}{2} \leqslant \lambda, \tau<1$ and

$$
K^{\frac{2}{3}+\delta}<G<K^{1-\delta} .
$$

The extension of the summation to $f \geqslant 3 K$ can be performed in view of (4.49) with an appropriate shift of the contour to the left.

This means that we have

$$
\begin{aligned}
& \sum_{f=1}^{\infty} \mathcal{H}_{1}\left(\lambda ; f ; h_{0}\right) f^{-\tau} \exp \left(-(f / K)^{\mu}\right) \\
& =-\frac{1}{2 \pi^{4} \mu} \zeta\left(\lambda+\frac{1}{2}\right)\left(\hat{h}_{0}\right)^{\prime}\left(\frac{1}{2}\right) \\
& \quad \times \int_{(3)} \zeta\left(w+\tau+\frac{1}{2}\right) \zeta(w+\lambda+\tau) K^{w} \Gamma\left(\frac{w}{\mu}\right) \mathrm{d} w \\
& \quad-\frac{1}{\pi^{2} \mu}(2 \pi)^{2(\lambda-1)} \sec (\pi \lambda) \zeta\left(\frac{3}{2}-\lambda\right) \hat{h}_{0}(1-\lambda) \\
& \quad \times \int_{(3)} \zeta\left(w+\tau+\frac{1}{2}\right) \zeta(w+1-\lambda+\tau) K^{w} \Gamma\left(\frac{w}{\mu}\right) \mathrm{d} w .
\end{aligned}
$$

Also,

$$
\begin{aligned}
& \sum_{f=1}^{\infty} \mathcal{H}_{1}\left(\lambda ; f ; h_{0}\right) f^{\tau-1} U_{0}(f K) \\
& =-\frac{1}{2 \pi^{4} \mu} \zeta\left(\lambda+\frac{1}{2}\right)\left(\hat{h}_{0}\right)^{\prime}\left(\frac{1}{2}\right) \\
& \quad \times \int_{(-3)} \zeta\left(\frac{3}{2}-\tau-w\right) \zeta(1+\lambda-\tau-w)\left(4 \pi^{2} / K\right)^{w} u_{0}(w) \Gamma\left(\frac{w}{\mu}\right) \mathrm{d} w \\
& \quad-\frac{1}{\pi^{2} \mu}(2 \pi)^{2(\lambda-1)} \sec (\pi \lambda) \zeta\left(\frac{3}{2}-\lambda\right) \hat{h}_{0}(1-\lambda) \\
& \quad \times \int_{(-3)} \zeta\left(\frac{3}{2}-\tau-w\right) \zeta(2-\lambda-\tau-w)\left(4 \pi^{2} / K\right)^{w} u_{0}(w) \Gamma\left(\frac{w}{\mu}\right) \mathrm{d} w
\end{aligned}
$$


Before specialising the above formula, note that

$$
\left(\hat{h}_{0}\right)^{\prime}\left(\frac{1}{2}\right)=2 i \pi^{\frac{3}{2}} K^{3} G+O\left(K G^{3}\right) .
$$

We then put $\lambda=\tau, \frac{1}{2}<\tau<1$. Then the right side of (4.60) is asymptotically equal to

$$
\frac{2}{\pi^{\frac{3}{2}}} \zeta^{2}\left(\tau+\frac{1}{2}\right) \zeta(2 \tau) K^{3} G
$$

and that of (4.61) to

$$
-\frac{2}{\pi^{\frac{3}{2}}} \zeta^{2}\left(\tau+\frac{1}{2}\right) K^{3} G\left(4 \pi^{2} / K\right)^{\frac{1}{2}-\tau} u_{0}\left(\frac{1}{2}-\tau\right) \Gamma\left(\frac{\frac{1}{2}-\tau}{\mu}\right) .
$$

Inserting these expressions into (4.60) we find that

$$
\mathcal{C}(\tau, \tau ; K, G)=(1+o(1)) \frac{2}{\pi^{\frac{3}{2}}} \zeta^{2}\left(\tau+\frac{1}{2}\right) \zeta(2 \tau) K^{3} G,
$$

which leads to (4.2). Namely, similarly as in [Iv9, eq. (7.10)-(7.11)], we note that we have

$$
\begin{aligned}
& \int_{K_{0}}^{2 K_{0}} \mathcal{C}(\tau, \tau ; K, G) \mathrm{d} K \\
& =\sum_{j \geqslant 1} \alpha_{j} H_{j}\left(\frac{1}{2}\right) H_{j}^{2}(\tau) \int_{K_{0}}^{2 K_{0}}\left(\kappa_{j}^{2}+\frac{1}{4}\right) \exp \left(-\left(\kappa_{j}-K\right)^{2} G^{-2}\right) \mathrm{d} K+O(1) \\
& =\sqrt{\pi} G \sum_{K_{0}<\kappa_{j} \leqslant 2 K_{0}} \alpha_{j} H_{j}\left(\frac{1}{2}\right) H_{j}^{2}(\tau) \kappa_{j}^{2}+o\left(K_{0}^{4} G\right) .
\end{aligned}
$$

On the other hand, from the main term on the right-hand side of (4.65) we obtain

$$
\begin{aligned}
& 2 \pi^{-3 / 2} \zeta^{2}\left(\tau+\frac{1}{2}\right) \zeta(2 \tau) \int_{K_{0}}^{2 K_{0}} K^{3} G \mathrm{~d} K \\
& =\frac{1}{2} G \pi^{-3 / 2} \zeta^{2}\left(\tau+\frac{1}{2}\right) \zeta(2 \tau)\left(\left(2 K_{0}\right)^{4}-K_{0}^{4}\right) .
\end{aligned}
$$

Here we take $G=K_{0}^{1-\varepsilon}$ say, then we replace $K_{0}$ by $K_{0} 2^{-\ell}$ and sum over $\ell \geqslant 1$, and finally replace $K_{0}$ by $K$ to obtain

$$
\sum_{\kappa_{j} \leqslant K} \alpha_{j} H_{j}\left(\frac{1}{2}\right) H_{j}^{2}(\tau) \kappa_{j}^{2}=\left(\frac{1}{2} \pi^{-2} \zeta^{2}\left(\tau+\frac{1}{2}\right) \zeta(2 \tau)+o(1)\right) K^{4}
$$

as $K \rightarrow \infty$. The desired formula (4.2) follows then by partial summation from (4.66). 
To prove (4.3), set $\lambda=\frac{1}{2}, \frac{1}{2}<\tau<1$. This case is treated in Section 3.3 of [Mo6], and we could appeal to Lemma 3.8 therein. But it is the same as to use (4.60) and (4.61) with this specialisation. Thus, the right side of (4.60) with $\lambda=\frac{1}{2}$ is equal to

$$
\begin{aligned}
& \frac{1}{2 \pi^{4} \mu} \int_{(3)}\left\{2\left(\hat{h}_{0}\right)^{\prime}\left(\frac{1}{2}\right)\left(\log (2 \pi)-c_{E}-\frac{\zeta^{\prime}}{\zeta}\left(w+\tau+\frac{1}{2}\right)\right)-\frac{1}{2}\left(\hat{h}_{0}\right)^{\prime \prime}\left(\frac{1}{2}\right)\right\} \\
& \times \zeta^{2}\left(w+\tau+\frac{1}{2}\right) K^{w} \Gamma\left(\frac{w}{\mu}\right) \mathrm{d} w
\end{aligned}
$$

and that of (4.61) to

$$
\begin{aligned}
& \frac{1}{2 \pi^{4} \mu} \int_{(-3)}\left\{2\left(\hat{h}_{0}\right)^{\prime}\left(\frac{1}{2}\right)\left(\log (2 \pi)-c_{E}-\frac{\zeta^{\prime}}{\zeta}\left(\frac{3}{2}-\tau-w\right)\right)-\frac{1}{2}\left(\hat{h}_{0}\right)^{\prime \prime}\left(\frac{1}{2}\right)\right\} \\
& \quad \times \zeta^{2}\left(\frac{3}{2}-\tau-w\right)\left(4 \pi^{2} / K\right)^{w} u_{0}(w) \Gamma\left(\frac{w}{\mu}\right) \mathrm{d} w
\end{aligned}
$$

where $c_{E}$ is the Euler constant, the $u_{0}$ is specialized accordingly, and

$$
\left(\hat{h}_{0}\right)^{\prime \prime}\left(\frac{1}{2}\right)=8 i \pi^{\frac{3}{2}} K^{3} G \log K+O\left(K G^{3} \log K\right) .
$$

Hence we have

$$
\mathcal{C}\left(\frac{1}{2}, \tau ; K, G\right)=(1+o(1)) \frac{4}{\pi^{\frac{3}{2}}} \zeta^{2}\left(\tau+\frac{1}{2}\right) K^{3} G \log K,
$$

which implies (4.3) by the procedure used in the previous case. This completes the proof of Theorem 2 .

\section{The asymptotics of the $\Lambda$-function}

We shall apply now Theorem 1 with a specific (Gaussian) exponential weight function, namely

$$
g(t)=\frac{1}{2 \sqrt{\pi} G}\left\{\exp \left(-\left(\frac{T-t}{G}\right)^{2}\right)+\exp \left(-\left(\frac{T+t}{G}\right)^{2}\right)\right\}
$$

which is a standard one, either in this or in a slightly changed form (without the factor $1 /(2 \sqrt{\pi} G))$. Obviously this choice of $g$ satisfies the basic assumption in Section 1.

The crucial thing needed in the estimation of $E_{2}(T, \sigma)$ and related quantities is the function $\Lambda(r ; \tau, g)$, defined by (2.8), and we proceed in this section to give 
its asymptotic evaluation. The main formula is (5.14), but we have found it more expedient to leave it in this form than to formulate a concrete theorem or lemma which would provide the needed asymptotic evaluation. The form that will be given in the sequel is sharper and more complete than the one that can be found in [Mo6, Chapter 5]. We suppose that the parameters $r, G$ satisfy

$$
1 \ll r \leqslant T G^{-1} \log ^{5} T, \quad T^{\varepsilon} \leqslant G \leqslant T^{1-\varepsilon},
$$

which are the relevant ranges for our investigations. The case $r<0$ is completely analogous, and the range for $r$ not covered by (5.2) is treated in [Mo6], where it is shown that the contribution is negligible. In the case of the weight function (5.1) (without the factor $1 /(2 \sqrt{\pi} G)$ ) we shall have

$$
g_{c}(x)=2 \sqrt{\pi} G \mathrm{e}^{-\frac{1}{4} G^{2} x^{2}} \cos (x T)
$$

However, to keep in tune with the notation of [Mo6], we omit $2 \sqrt{\pi} G$ in subsequent calculations. Moreover, the exponential factor in (5.3) shows that the contribution of $y>G^{-1} \log T$ in (3.57) is negligible, so that by changing $y$ to $1 / y$ it is sufficient to start with the evaluation of the integral

$$
\begin{aligned}
I:= & \int_{0}^{G^{-1} \log T} y^{2 \tau-3 / 2}(1+y)^{-\tau} \cos (T \log (1+y)) \exp \left(-\frac{1}{4} G^{2} \log ^{2}(1+y)\right) \\
& \times \operatorname{Re}\left\{y^{i r} \frac{\Gamma^{2}\left(\frac{1}{2}+i r\right)}{\Gamma(1+2 i r)} F\left(\frac{1}{2}+i r, \frac{1}{2}+i r ; 1+2 i r ;-y\right)\right\} \mathrm{d} y,
\end{aligned}
$$

where $\tau \geqslant \frac{1}{2}(\tau \neq 1)$ is a given constant, and of course $I$ depends on $T, r, G$ and $\tau$. There are several ways to evaluate $I$ asymptotically, but the simplest procedure seems to use the following quadratic transformation formula (see [L, eq. (9.6.12)]), which is valid if $|\arg (1-z)|<\pi, 2 \beta \neq-1,-3,-5, \ldots$ :

$$
\begin{aligned}
F(\alpha, \beta ; 2 \beta ; z)= & \left(\frac{1+\sqrt{1-z}}{2}\right)^{-2 \alpha} \\
& \times F\left(\alpha, \alpha-\beta+\frac{1}{2} ; \beta+\frac{1}{2} ;\left(\frac{1-\sqrt{1-z}}{1+\sqrt{1-z}}\right)^{2}\right) .
\end{aligned}
$$

Then the relevant part of $I$ becomes

$$
\begin{aligned}
& \int_{0}^{G^{-1} \log T} y^{2 \tau-3 / 2}(1+y)^{-\tau} \cos (T \log (1+y)) \exp \left(-\frac{1}{4} G^{2} \log ^{2}(1+y)\right) \\
& \quad \times \operatorname{Re}\left\{y^{i r} \frac{\Gamma^{2}\left(\frac{1}{2}+i r\right)}{\Gamma(1+2 i r)}\left(\frac{1+\sqrt{1+y}}{2}\right)^{-1-2 i r}\right. \\
& \left.\quad \times F\left(\frac{1}{2}+i r, \frac{1}{2} ; 1+i r ;\left(\frac{1-\sqrt{1+y}}{1+\sqrt{1+y}}\right)^{2}\right)\right\} \mathrm{d} y .
\end{aligned}
$$


We recall (2.9), and insert it in (5.6) with $\alpha=\frac{1}{2}+i r, \beta=\frac{1}{2}, \gamma=1+i r$,

$$
z=\left(\frac{1-\sqrt{1+y}}{1+\sqrt{1+y}}\right)^{2} \ll G^{-2} \log ^{2} T=o(1) \quad(T \rightarrow \infty),
$$

since $0 \leqslant y \leqslant G^{-1} \log T$ in (5.6). Note that, for $k \geqslant 1$,

$$
\left|\frac{\left(\frac{1}{2}+i r\right)_{k}}{(1+i r)_{k}}\right| \leqslant 1, \quad \frac{\left(\frac{1}{2}+i r\right)_{k}}{(1+i r)_{k}}=\left(\frac{i r}{k+i r}\right)^{1 / 2}\left(1+O\left(\frac{1}{r}\right)\right)
$$

uniformly in $k$, with an appropriate choice of branch. Therefore the main contribution to $I$ will come from the constant term (i.e., unity) in the series expansion (2.9), while the remaining terms will be of a similar nature, only of a lower order of magnitude. The series can be truncated in such a way that the tails will make a negligible contribution; this procedure will be repeatedly used without further explicit mention in subsequent calculations. For example, we develop into series the terms $(1+y)^{-\tau}$ and $\left(\frac{1+\sqrt{1+y}}{2}\right)^{-1}$, noting that the main contribution will again come from the constant term unity. Now we use Stirling's formula for the gamma-function in the form $\left(t \geqslant t_{0}>0,0 \leqslant \sigma \leqslant 1\right)$

$$
\Gamma(s)=\sqrt{2 \pi} t^{\sigma-\frac{1}{2}} \exp \left(-\frac{1}{2} \pi t+i t \log t-i t+\frac{1}{2} \pi i\left(\sigma-\frac{1}{2}\right)\right) \cdot\left(1+O_{\sigma}\left(t^{-1}\right)\right),
$$

with the understanding that the $O$-term in (5.7) admits an asymptotic expansion in terms of negative powers of $t$. Therefore we have

$$
\frac{\Gamma^{2}\left(\frac{1}{2}+i r\right)}{\Gamma(1+2 i r)}=\sqrt{\pi} r^{-1 / 2} \mathrm{e}^{-2 i r \log 2-\frac{1}{4} \pi i} \cdot\left(1+O\left(\frac{1}{r}\right)\right)
$$

for the gamma-factors in (5.6), where the $O$-term admits an asymptotic expansion. In this way the problem is reduced to the evaluation of the integral

$$
\begin{aligned}
& \sqrt{\pi} r^{-1 / 2} \int_{0}^{G^{-1} \log T} y^{2 \tau-3 / 2} \cos (T \log (1+y)) \exp \left(-\frac{1}{4} G^{2} \log ^{2}(1+y)\right) \\
& \times \operatorname{Re}\left\{y^{i r} \exp \left(-2 i r \log 2-2 i r \log \left(\frac{1+\sqrt{1+y}}{2}\right)-\frac{1}{4} \pi i\right)\right\} \mathrm{d} y \\
& =\sqrt{\pi} r^{-1 / 2} \int_{0}^{G^{-1} \log T} y^{2 \tau-3 / 2} \cos (T \log (1+y)) \exp \left(-\frac{1}{4} G^{2} \log ^{2}(1+y)\right) \\
& \times \cos \left(r \log y-r \log 4-2 r \log \left(\frac{1+\sqrt{1+y}}{2}\right)-\frac{1}{4} \pi\right) \mathrm{d} y,
\end{aligned}
$$

But as

$$
\cos \alpha \cos \beta=\frac{1}{2}[\cos (\alpha+\beta)+\cos (\alpha-\beta)],
$$


we have in fact to consider

$$
\sqrt{\pi} r^{-1 / 2} \mathrm{e}^{-i r \log 4} \int_{0}^{G^{-1} \log T} y^{2 \tau-3 / 2} \exp \left(-\frac{1}{4} G^{2} \log ^{2}(1+y)\right) \mathrm{e}^{i \mathcal{F}_{ \pm}(y, r)-\frac{1}{4} i \pi} \mathrm{d} y,
$$

with

$$
\mathcal{F}_{ \pm}(y ; r, T):=r \log y-2 r \log \left(\frac{1+\sqrt{1+y}}{2}\right) \pm T \log (1+y),
$$

so that

$$
\frac{\partial \mathcal{F}_{ \pm}(y ; r, T)}{\partial y}=\frac{r}{y}-\frac{r}{1+y+\sqrt{1+y}} \pm \frac{T}{1+y} .
$$

Note that in our range for $y$, which is $0<y \leqslant G^{-1} \log T$, the derivative of $\mathcal{F}_{+}$is positive, so there will be no saddle point. Hence we shall discuss in detail only the more difficult case of $\mathcal{F}_{-}$(henceforth denoted by $\mathcal{F}$ ), which has a saddle point $y_{0}$, the root of

$$
\frac{r}{y}-\frac{r}{1+y+\sqrt{1+y}}=\frac{T}{1+y} .
$$

This is equivalent to $T^{2} y^{2}-r^{2} y-r^{2}=0$, giving

$$
y_{0}=\frac{r}{T}\left(\sqrt{1+\frac{r^{2}}{4 T^{2}}}+\frac{r}{2 T}\right)
$$

so that $y_{0} \sim r / T$ as $T \rightarrow \infty$. Then

$$
\mathcal{F}\left(y_{0}\right)=r \log y_{0}-2 r \log \left(\frac{1+\sqrt{1+y_{0}}}{2}\right)-T \log \left(1+y_{0}\right) .
$$

Using (5.11) a calculation gives

$$
\begin{aligned}
r \log y_{0} & =r \log \frac{r}{T}+\frac{r^{2}}{2 T}+O\left(\frac{r^{4}}{T^{3}}\right), \\
-2 r \log \left(\frac{1+\sqrt{1+y_{0}}}{2}\right) & =-\frac{r^{2}}{2 T^{2}}-\frac{r^{3}}{16 T^{2}}+O\left(\frac{r^{4}}{T^{3}}\right), \\
-T \log \left(1+y_{0}\right) & =-r+\frac{r^{3}}{24 T^{2}}+O\left(\frac{r^{4}}{T^{3}}\right),
\end{aligned}
$$

and the $O$-terms admit an asymptotic expansion in powers of $r / T$. Therefore we obtain

$$
\mathcal{F}\left(y_{0}\right)-r \log 4=r \log \left(\frac{r}{4 \mathrm{e} T}\right)+\sum_{j=3}^{N} c_{j} r^{j} T^{1-j}+O_{N}\left(r^{N+1} T^{-N}\right)
$$


for any given integer $N \geqslant 3$ and some effectively computable real constants $c_{j}\left(c_{3}=-1 / 48\right)$. As

$$
\mathcal{F}^{\prime \prime}\left(y_{0}\right) \sim-\frac{T^{2}}{r} \quad(T \rightarrow \infty),
$$

it follows that the dominant contribution to $I$ is a multiple of

$$
\begin{aligned}
T^{\frac{1}{2}-2 \tau} r^{2 \tau-\frac{3}{2}} \exp \left\{-\frac{1}{4} G^{2} \log ^{2}\left(1+y_{0}\right)+i \mathcal{F}\left(y_{0}\right)-i r \log 4\right\} \\
=T^{\frac{1}{2}-2 \tau} r^{2 \tau-\frac{3}{2}} \\
\quad \times \exp \left\{-\frac{1}{4} G^{2} \log ^{2}\left(1+y_{0}\right)+i r \log \left(\frac{r}{4 \mathrm{e} T}\right)+i \sum_{j=3}^{N} c_{j} r^{j} T^{1-j}+O_{N}\left(r^{N+1} T^{-N}\right)\right\} .
\end{aligned}
$$

This is understood in the following sense: the remaining terms in the evaluation of $I$ are either negligible, or similar in nature to (5.14) (meaning that the oscillating exponential factor is the same, which is crucial), only of the lower order of magnitude than (5.14). We shall show now briefly show how the saddle point method does indeed lead to this assertion.

To see this we turn back to the integral in (5.9). We use the techniques which were used in establishing (7.1.30) and (7.1.31) of [Mo6]. With $y_{0}$ as in (5.11) we have that the relevant integral is equal to

$$
y_{0} \mathrm{e}^{-\pi i / 4} \int_{-\xi_{0}}^{\xi_{0}} f_{0}(\xi) \mathrm{e}^{i f(\xi)} \mathrm{d} \xi \quad\left(\xi_{0}=r^{\varepsilon-1 / 2}\right),
$$

plus as error term which is $\ll_{\varepsilon} \exp \left(-r^{\varepsilon}\right)$. This error term is negligible if

$$
r \geqslant(\log T)^{C(\varepsilon)}
$$

with $C(\varepsilon)(>0)$ sufficiently large. The functions appearing in (5.15) are ( $\xi$ is the variable of integration)

$$
\begin{aligned}
f_{0}(\xi) & :=y^{2 \tau-3 / 2} \exp \left(-\frac{1}{4} G^{2} \log ^{2}(1+y)\right), \quad y:=y_{0}+y_{0} \xi \mathrm{e}^{-\pi i / 4}, \\
f(\xi) & :=\mathcal{F}_{-}(\xi ; r, T)=r \log y-2 r \log \left(\frac{1+\sqrt{1+y}}{2}\right)-T \log (1+y),
\end{aligned}
$$

where we assume that (5.1) and (5.16) hold. This enables us to replace $f_{0}(\xi)$ with

$$
y_{0}^{2 \tau-3 / 2} \exp \left(-\frac{1}{4} G^{2} \log ^{2}\left(1+y_{0}\right)\right),
$$

on expanding $f_{0}(\xi)$ into its Taylor series at $y_{0}$. Likewise, since $\mathcal{F}^{\prime}\left(y_{0}\right)=0$,

$$
f(\xi)=\mathcal{F}\left(y_{0}+y_{0} \xi \mathrm{e}^{-\pi i / 4}\right)=\mathcal{F}\left(y_{0}\right)+\frac{1}{2} i y_{0}^{2} \xi^{2}\left(-\mathcal{F}^{\prime \prime}\left(y_{0}\right)\right)+G\left(\xi ; r, T, y_{0}\right),
$$


say, where $G$ can be expanded into Taylor series and

$$
G\left(\xi ; r, T, y_{0}\right) \ll y_{0}^{3} \xi_{0}^{3} r y_{0}^{-3}=r^{3 \varepsilon-1 / 2} .
$$

After this the ensuing integrals are evaluated by using the formula (proved by induction on $k$ )

$$
\int_{-\Xi_{0}}^{\Xi_{0}} \xi^{2 k} \mathrm{e}^{-\frac{1}{2} c \xi^{2}} \mathrm{~d} \xi=2^{k+\frac{1}{2}} \Gamma\left(k+\frac{1}{2}\right) c^{-\frac{1}{2}-k}+O_{k}\left(c^{-1} \Xi_{0}^{k-1} \mathrm{e}^{-\frac{1}{2} c \Xi_{0}^{2}}\right),
$$

provided that

$$
k=0,1,2, \ldots, c>0, \Xi_{0}>0, \Xi_{0} \sqrt{c} \geqslant 1 .
$$

In our case

$$
c=-y_{0}^{2} F^{\prime \prime}\left(y_{0}\right)>0, \Xi_{0}=r^{\varepsilon-1 / 2}, \Xi_{0} \sqrt{c} \asymp r^{\varepsilon},
$$

so that (5.19) is satisfied. Collecting all the estimates, we see that the major contribution to $I$ is indeed furnished by (5.14).

In the case when the integral in (5.9) has no saddle point, i.e., the case of $\mathcal{F}_{+}$, we turn the segment of integration by the angle $r^{-1 / 2}$, say, to obtain that the contribution of the integral is in this case negligible.

In the case when (5.16) fails, more precisely when

$$
|r| \leqslant(\log T)^{C(\varepsilon)}
$$

we apply the technique of [Mo6, Lemma 5.2], to see that the integral in question in the above range is $\ll T^{\frac{1}{2}-2 \tau}$, which is sufficiently sharp for our purposes.

\section{The weighted fourth moment when $\frac{1}{2}<\sigma<\frac{3}{4}$}

With the use of Theorem 1 and the asymptotics of Section 5 we can derive the explicit formula for the fundamental function

$$
\begin{aligned}
I_{2}(T, \tau, G):= & \frac{1}{\sqrt{\pi} G} \int_{-\infty}^{\infty}|\zeta(\tau+i t+i T)|^{4} \mathrm{e}^{-(t / G)^{2}} \mathrm{~d} t \\
& \left(\frac{1}{2}<\tau<\frac{3}{4}, T^{1 / 3+\varepsilon} \leqslant G \leqslant T^{1-\varepsilon}\right) .
\end{aligned}
$$

This formula, as in the case when $\tau=1 / 2$ (see [I2], [Mo6]), can be integrated over $T$. It will then lead to explicit results on the function $E_{2}(T, \sigma)$, the error term in the asymptotic formula for $\int_{0}^{T}|\zeta(\sigma+i t)|^{4} \mathrm{~d} t$. Our result on $I_{2}(T, \sigma)$ and its integral is given by 
Theorem 3. If $I_{2}(T, \sigma, G)$ is given by (6.1), $\frac{1}{2}<\sigma<\frac{3}{4}, T^{1 / 3+\varepsilon} \leqslant G \leqslant T^{1-\varepsilon}$, $Y_{0}=\left(\kappa_{j} / T\right)\left(\sqrt{1+\left(\kappa_{j} / 4 T\right)^{2}}+\kappa_{j} /(2 T)\right)$, then we have

$$
\begin{aligned}
& I_{2}(T, \sigma, G) \sim O(1) \\
& \quad+C(\sigma) T^{\frac{1}{2}-2 \sigma} \sum_{\kappa_{j} \leqslant T G^{-1} \log T} \alpha_{j} \kappa_{j}^{2 \sigma-3 / 2} H_{j}^{2}\left(\frac{1}{2}\right) H_{j}\left(2 \sigma-\frac{1}{2}\right) \mathrm{e}^{-\frac{1}{4} G^{2} \log ^{2}\left(1+Y_{0}\right)} \\
& \quad \times \sin \left(\kappa_{j} \log \frac{\kappa_{j}}{4 \mathrm{e} T}+c_{3} \kappa_{j}^{3} T^{-2}\right) .
\end{aligned}
$$

We also have, for $\bar{Y}_{0}=\left(\kappa_{j} / V\right)\left(\sqrt{1+\left(\kappa_{j} / 4 V\right)^{2}}+\kappa_{j} /(2 V)\right)$, and $V^{1 / 3+\varepsilon} \leqslant G \leqslant$ $V^{1-\varepsilon}(D>0)$,

$$
\begin{aligned}
& \int_{0}^{V} I_{2}(T, \sigma ; G) \mathrm{d} T \sim \frac{\zeta^{4}(2 \sigma)}{\zeta(4 \sigma)} V+\frac{V}{3-4 \sigma}\left(\frac{V}{2 \pi}\right)^{2-4 \sigma} \frac{\zeta^{4}(2-2 \sigma)}{\zeta(4-4 \sigma)} \\
& \quad+V^{2-2 \sigma}\left(a_{0}(\sigma)+a_{1}(\sigma) \log V+a_{2}(\sigma) \log ^{2} V\right)+ \\
& \quad+C(\sigma) V^{\frac{3}{2}-2 \sigma} \sum_{\kappa_{j} \leqslant V G^{-1} \log T} \alpha_{j} \kappa_{j}^{2 \sigma-5 / 2} H_{j}^{2}\left(\frac{1}{2}\right) H_{j}\left(2 \sigma-\frac{1}{2}\right) \mathrm{e}^{-\frac{1}{4} G^{2} \log ^{2}\left(1+\bar{Y}_{0}\right)} \\
& \quad \times \cos \left(\kappa_{j} \log \left(\frac{\kappa_{j}}{4 e V}\right)+c_{3} \kappa_{j}^{3} V^{-2}\right)+O(G)+O\left(V^{1 / 3} \log ^{D} V\right)
\end{aligned}
$$

with suitable constants $C(\sigma), C_{1}(\sigma)$, and $a_{j}(\sigma)$, which may be explicitly evaluated. The meaning of the symbol $\sim$ is that besides the spectral sums in (6.2)-(6.3) a finite number of other sums are to appear, each of which is similar in nature to the the corresponding sum above, but of a lower order of magnitude.

Proof of Theorem 3. The meaning of the symbol $\sim$ was already explained after (5.14). Each of the omitted sums is either negligibly small, or similar in structure to the ones appearing above, namely it has the same oscillatory factors as the corresponding sums above. When estimated, their contribution will be (by a power of $T$ or $V$ ) smaller than the contribution of the sums in (6.2) and (6.3).

To prove Theorem 3, we use (2.3)-(2.8) of Theorem 1. The derivation of (6.2) is similar to the proof of Theorem 5.2 of [I2] or Theorem 5.1 of [Mo6], starting from the spectral decomposition of $\mathcal{L}(g ; \tau, \tau)$ when $\tau=1 / 2$. Thus we shall be relatively brief, noting that the sum in (6.2) comes from the discrete spectral part (2.5) and (5.14). We shall need (5.14) with $\tau=\sigma, \frac{1}{2}<\sigma<1$. The weight function $g$ will be (5.1), hence

$$
g_{c}(x)=\mathrm{e}^{-\frac{1}{4} G^{2} x^{2}} \cos (x T) .
$$

In view of the expressions for $M_{\ell}^{*}(\ell=0, \ldots, 6)$ (see (3.74)-(3.89)) of the main term (cf. $\mathcal{Z}_{r}(\tau, g)$ in (2.4)) will be $\mathrm{O}(1)$, as will also be the contribution of $\mathcal{Z}_{h}(\tau, g)$ in (2.7). The contribution of $\mathcal{Z}_{c}(\tau, g)$, given by the integral in (2.6), is estimated 
by the use of (5.14). It will be $O(1)$ plus the term which is

$$
\begin{aligned}
& \ll \log ^{2} T \int_{-T G^{-1} \log T}^{T G^{-1} \log T}\left|\zeta\left(\frac{1}{2}+i r\right)\right|^{4}\left|\zeta\left(2 \sigma-\frac{1}{2}\right)\right|^{2} T^{1 / 2-2 \sigma}(|r|+1)^{2 \sigma-3 / 2} \mathrm{~d} r \\
& \ll\left(T G^{-1}\right)^{\frac{5}{4}+2 \sigma-\frac{3}{2}} T^{\frac{1}{2}-2 \sigma} \log ^{C} T \\
& =T^{1 / 4} G^{1 / 4-2 \sigma} \log ^{C} T \leqslant 1
\end{aligned}
$$

for $G \geqslant T^{1 / 3}$, since $\sigma>\frac{1}{2}$. Here we used the trivial bound $1 /\left|\zeta\left(\frac{1}{2}+i t\right)\right| \ll \log |t|$, coupled with the Cauchy-Schwarz inequality for integrals and the bounds (see [I1])

$$
\int_{0}^{T}\left|\zeta\left(\frac{1}{2}+i t\right)\right|^{8} \mathrm{~d} t \ll T^{3 / 2} \log ^{C} T, \quad \int_{1}^{T}|\zeta(\tau+i t)|^{4} \mathrm{~d} t \ll T \quad\left(\frac{1}{2}<\tau \leqslant 1\right) .
$$

To prove (6.3), we integrate first the spectral decomposition of Theorem 1 from $V$ to $2 V$, eventually replacing $V$ by $V 2^{-j}$ and summing over $j \in \mathbb{N}$. When we apply (5.14) and integrate, we essentially have to integrate $T^{\frac{1}{2}-2 \tau-i r}$ over $T$, which accounts for the increase in order of $T / \kappa_{j}$ in (6.3), and one can check that integration will transform the sine into cosine. Here care should be exerted when one computes the main term on the right-hand side of (6.3). This is given (cf. $\left.M\left(\mathrm{p}_{\tau} ; g\right)\right)$ by eqs.(3.74)-(3.89). In the evaluation we make repeatedly use of the formula (see [I2, Section 5.1])

$$
\frac{\Gamma^{(k)}(s)}{\Gamma(s)}=\sum_{j=0}^{k} b_{j, k}(s) \log ^{j} s+c_{-1, k} s^{-1}+\ldots+c_{-r, k} s^{-r}+O_{r}\left(|s|^{-r-1}\right)
$$

for any fixed integers $k \geqslant 1, r \geqslant 0$, where each $b_{j, k}\left(\sim b_{j, k}\right.$ for a suitable constant $b_{j, k}$ ) has an asymptotic expansion in non-positive powers of $s$. It transpires that one encounters integrals of the type

$$
\begin{aligned}
& \frac{1}{\sqrt{\pi G}} \int_{-\infty}^{\infty} \log ^{r}\left(\frac{1}{2}+i T+i t\right) \mathrm{e}^{-(t / G)^{2}} \mathrm{~d} t \\
& =\frac{1}{\sqrt{\pi}} \int_{-\infty}^{\infty} \log ^{r}\left(\frac{1}{2}+i T+i u G\right) \mathrm{e}^{-u^{2}} \mathrm{~d} u \\
& =\frac{1}{\sqrt{\pi}} \int_{-\log T}^{\log T} \log ^{r}\left(\frac{1}{2}+i T+i u G\right) \mathrm{e}^{-u^{2}} \mathrm{~d} u+O_{A}\left(T^{-A}\right),
\end{aligned}
$$

for any fixed $A>0$. For $|u| \leqslant \log T$ one has the power series expansion

$$
\begin{aligned}
& \log ^{r}\left(\frac{1}{2}+i T+i u G\right)=\log ^{r}(i T) \\
& \quad+\sum_{k=1}^{r}\left(\begin{array}{l}
r \\
k
\end{array}\right)(\log i T)^{r-k}\left(\frac{u G}{T}+\frac{1}{2 i T}-\frac{1}{2}\left(\frac{u G}{T}+\frac{1}{2 i T}\right)^{2}+\ldots\right)^{k}
\end{aligned}
$$


which is inserted in (6.6). The evaluation is completed by applying (5.18). The main term in (6.3) is the same one as in (6.2) (with $V$ replacing $T$ ), and the constant standing in front of the term $T^{3-4 \sigma}$ was first explicitly evaluated by Kačènas [K1], [K2]. The contributions of $\mathcal{Z}_{h}(\tau, g)$ and $\mathcal{Z}_{c}(\tau, g)$ will be absorbed by the error terms after integration. This ends our discussion of Theorem 3.

Next, we consider $E_{2}\left(T, \frac{3}{4}\right)$ by using (3.88)-(3.90). We obtain, with suitable constants $A_{j}$, which may be explicitly evaluated,

$$
\begin{aligned}
& \int_{0}^{T}\left|\zeta\left(\frac{3}{4}+i t\right)\right|^{4} \mathrm{~d} t=\frac{\zeta^{4}\left(\frac{3}{2}\right)}{\zeta(3)} T \\
& \quad+T^{1 / 2}\left(A_{0}+A_{1} \log T+A_{2} \log ^{2} T\right)+E_{2}\left(T, \frac{3}{4}\right)
\end{aligned}
$$

with

$$
E_{2}\left(T, \frac{3}{4}\right) \ll T^{1 / 2} \log ^{3} T .
$$

Note that the bound (6.8) for the error term is, by a log-factor, larger than the order of the second main term in (6.7). Indeed, it is very plausible that the bound (6.8) is far from the truth and that we have

$$
E_{2}(T, \sigma) \ll_{\varepsilon} T^{3 / 2-2 \sigma+\varepsilon} \quad\left(\frac{1}{2}<\sigma<\frac{3}{4}\right)
$$

and

$$
E_{2}(T, \sigma) \ll_{\varepsilon} T^{\varepsilon} \quad\left(\frac{3}{4} \leqslant \sigma<1\right) .
$$

Here and later $\varepsilon$ denotes arbitrarily small, positive constants, not necessarily the same ones at each occurrence, and $f \ll_{\varepsilon} g$ means that the $\ll$-constant depends on $\varepsilon$. Also note that $C$ will denote a generic positive constant.

The conjectures (6.9)-(6.10) were made in [I6]. They are very strong, since they imply that $\zeta\left(\frac{1}{2}+i t\right) \ll_{\varepsilon}|t|^{1 / 8+\varepsilon}$ and $\zeta(\sigma+i t) \ll_{\varepsilon}|t|^{\varepsilon}$ for $\sigma \geqslant \frac{3}{4}$. They are the analogues of the conjectures for the true order of the error term $E_{1}(T, \sigma)$ in (1.5) (see [Ma]). What seems possible to prove at present for the range $\frac{3}{4} \leqslant \sigma<1$ is (cf. [I6, Th. 2])

$$
\int_{0}^{T}|\zeta(\sigma+i t)|^{4} \mathrm{~d} t=\frac{\zeta^{4}(2 \sigma)}{\zeta(4 \sigma)} T+O\left(T^{2-2 \sigma} \log ^{3} T\right),
$$

which is far from the conjectured bound (6.10). In view of (6.11) there seems to be no point in further estimation of $E_{2}(T, \sigma)$ when $\frac{3}{4}<\sigma<1$, since the bounds that seem obtainable from the spectral decomposition are weaker than (6.11). When $\sigma=1$ we have (see [I3])

$$
\int_{1}^{T}|\zeta(1+i t)|^{4} \mathrm{~d} t=\frac{\zeta^{4}(2)}{\zeta(4)} T+O\left(\log ^{4} T\right),
$$

so that this case is covered, too $\left(\zeta^{4}(2) / \zeta(4)=\pi^{2} / 72\right)$. 


\section{The fourth moment when $\frac{1}{2}<\sigma<\frac{3}{4}$}

We have prepared the groundwork for the results on $E_{2}(T, \sigma)$, the error term for the fourth moment off the critical line (see (1.6)), in the previous sections. Now we can proceed with the statement of our results.

Teorem 4. If $\sigma$ is a fixed number such that $\frac{1}{2}<\sigma<\frac{3}{4}$, and $E_{2}(T, \sigma)$ is defined by $(1.6)$, then with suitable constants $a_{j}(\sigma)$ we have

$$
\begin{aligned}
\int_{0}^{T}|\zeta(\sigma+i t)|^{4} \mathrm{~d} t= & \frac{\zeta^{4}(2 \sigma)}{\zeta(4 \sigma)} T+\frac{T}{3-4 \sigma}\left(\frac{T}{2 \pi}\right)^{2-4 \sigma} \frac{\zeta^{4}(2-2 \sigma)}{\zeta(4-4 \sigma)} \\
& +T^{2-2 \sigma}\left(a_{0}(\sigma)+a_{1}(\sigma) \log T+a_{2}(\sigma) \log ^{2} T\right)+E_{2}(T, \sigma)
\end{aligned}
$$

where with some $C>0$

$$
E_{2}(T, \sigma) \ll T^{2 /(1+4 \sigma)} \log ^{C} T \quad\left(\frac{1}{2}<\sigma<\frac{3}{4}\right) .
$$

Moreover,

$$
E_{2}(T, \sigma)=\Omega_{ \pm}\left(T^{\frac{3}{2}-2 \sigma}\right) \quad\left(\frac{1}{2}<\sigma<\frac{3}{4}\right) .
$$

More precisely, there exist constants $A=A(\sigma)>1$ and $B=B(\sigma)>0$ such that, for $T \geqslant T_{0}(\sigma)$, every interval $[T, A T]$ contains points $t_{1}=t_{1}(\sigma)$ and $t_{2}=t_{2}(\sigma)$ such that

$$
E_{2}\left(t_{1}, \sigma\right)>B t_{1}^{\frac{3}{2}-2 \sigma}, \quad E_{2}\left(t_{1}, \sigma\right)<-B t_{1}^{\frac{3}{2}-2 \sigma} \quad\left(\frac{1}{2}<\sigma<\frac{3}{4}\right) .
$$

Remarks. As usual, $f(x)=\Omega_{ \pm}(g(x))$ means that we have $\limsup _{x \rightarrow \infty} f(x) / g(x)>$ 0 and $\liminf _{x \rightarrow \infty} f(x) / g(x)<0$ for a given $g(x)>0\left(x \geqslant x_{0}\right)$. Note that $3-4 \sigma>2 /(1+4 \sigma)$ for $\frac{1}{2}<\sigma<\frac{1+\sqrt{2}}{4}$ and that $2-2 \sigma>2 /(1+4 \sigma)$ for $\sigma<\frac{3}{4}$. Thus our bound for the error term $E_{2}(T, \sigma)$ is already larger than the second main term in (1.6) unless $\frac{1}{2}<\sigma<\frac{1+\sqrt{2}}{4}$, but the bound in question is probably much too large (recall the conjectural bounds (6.9)-(6.10) for the order of $\left.E_{2}(T, \sigma)\right)$.

Theorem 5. Let $E_{2}(T, \sigma)$ be given by (1.6). If $\sigma$ is a fixed number such that $\frac{1}{2}<\sigma<\frac{3}{4}$, then for suitable $C=C(\sigma)>0$ we have

$$
\int_{0}^{T}\left|E_{2}(t, \sigma)\right|^{4 \sigma} \mathrm{d} t \ll T^{2} \log ^{C} T .
$$

We also have, for any constant $A \geqslant 1$,

$$
\int_{0}^{T}\left|E_{2}(t, \sigma)\right|^{A} \mathrm{~d} t \gg T^{1+A\left(\frac{3}{2}-2 \sigma\right)} .
$$


Note that when $\sigma=\frac{1}{2},(7.5)$ reduces to

$$
\int_{0}^{T} E_{2}^{2}(t) \mathrm{d} t \ll T^{2} \log ^{C} T
$$

where $E_{2}(T)=E_{2}\left(T, \frac{1}{2}\right)$ is the error term in the formula for the fourth moment of $\left|\zeta\left(\frac{1}{2}+i t\right)\right|$. The bound (7.7) is the sharpest one known (see [IM2], [Mo6]) and essentially best possible, since we have (see [I7])

$$
\int_{0}^{T} E_{2}^{2}(t) \mathrm{d} t \gg T^{2}
$$

The lower bound in (7.6), when $A=2, \sigma=\frac{1}{2}$, reduces to (7.7). Note that the conjecture (6.9) would furnish the upper bound

$$
\int_{0}^{T}\left|E_{2}(t, \sigma)\right|^{A} \mathrm{~d} t \ll_{\varepsilon} T^{1+A\left(\frac{3}{2}-2 \sigma\right)+\varepsilon},
$$

which is (up to ' $\varepsilon$ ') the same as the lower bound (7.6). The upper bound in (7.5), on the other hand, is much weaker than (6.9). This reflects, in general, the situation with $E_{2}(T, \sigma)$ : as $\sigma$ increases from $\frac{1}{2}$ to $\frac{3}{4}$, the quality of the bounds (either pointwise or in the mean square sense) decreases. The same phenomenon also occurs with bounds for the mean square of $\zeta(s)$ off the critical line (see $[\mathrm{Ma}]$ ).

Finally we remark that, by Hölder's inequality for integrals, (7.5) implies the mean square bound

$$
\int_{0}^{T} E_{2}^{2}(t, \sigma) \mathrm{d} t \ll T^{1+1 /(2 \sigma)} \log ^{C} T
$$

which may be compared to (7.7).

\section{Proof of the bounds when $\frac{1}{2}<\sigma<\frac{3}{4}$}

In this section we shall prove Theorem 4 and Theorem 5 , formulated in the preceding section. First we prove (7.1)-(7.2). We rewrite (6.3) of Theorem 3 as

$$
\int_{0}^{T} I_{2}(t, \sigma ; G) \mathrm{d} t=M(T, \sigma)+S(T, \sigma ; G)+R(T, \sigma ; G),
$$

say, where the main term is

$$
\begin{aligned}
M(T, \sigma):= & \frac{\zeta^{4}(2 \sigma)}{\zeta(4 \sigma)} T+\frac{T}{3-4 \sigma}\left(\frac{T}{2 \pi}\right)^{2-4 \sigma} \frac{\zeta^{4}(2-2 \sigma)}{\zeta(4-4 \sigma)} \\
& +T^{2-2 \sigma}\left(a_{0}(\sigma)+a_{1}(\sigma) \log T+a_{2}(\sigma) \log ^{2} T\right), \\
S(T, \sigma ; G):= & C(\sigma) T^{\frac{3}{2}-2 \sigma} \sum_{j=1}^{\infty} \alpha_{j} \kappa_{j}^{2 \sigma-5 / 2} H_{j}^{2}\left(\frac{1}{2}\right) \\
& \times H_{j}\left(2 \sigma-\frac{1}{2}\right) \mathrm{e}^{-\frac{1}{4} G^{2} \log ^{2}\left(1+Y_{0}\right)} \cos \left(\kappa_{j} \log \left(\frac{\kappa_{j}}{4 e T}\right)+c_{3} \kappa_{j}^{3} T^{-2}\right)
\end{aligned}
$$


is the spectral part, and the rest (error term) is

$$
R(T, \sigma ; G):=O(G)+O\left(T^{1 / 3} \log ^{D} T\right)
$$

We suppose that $T^{1 / 3+\varepsilon} \leqslant G=G(T) \leqslant T^{1-\varepsilon}$ and put first in (8.1)

$$
T_{1}=T-G \log T, T_{2}=2 T+G \log T
$$

Then

$$
\begin{aligned}
& \int_{T_{1}}^{T_{2}} I_{2}(t, \sigma ; G) \mathrm{d} t=\int_{-\infty}^{\infty}|\zeta(\sigma+i u)|^{4}\left(\frac{1}{\sqrt{\pi} G} \int_{T_{1}}^{T_{2}} \mathrm{e}^{-(t-u)^{2} / G^{2}} \mathrm{~d} t\right) \mathrm{d} u \\
& \geqslant\left.\int_{T}^{2 T} \zeta(\sigma+i u)\right|^{4}\left(\frac{1}{\sqrt{\pi} G} \int_{T-G \log T}^{2 T+G \log T} \mathrm{e}^{-(t-u)^{2} / G^{2}} \mathrm{~d} t\right) \mathrm{d} u
\end{aligned}
$$

But for $T \leqslant u \leqslant 2 T$ we have, by the change of variable $t-u=G v$,

$$
\begin{aligned}
& \frac{1}{\sqrt{\pi} G} \int_{T-G \log T}^{2 T+G \log T} \mathrm{e}^{-(t-u)^{2} / G^{2}} \mathrm{~d} t=\frac{1}{\sqrt{\pi}} \int_{(T-u) / G-\log T}^{(2 T+u) / G \log T} \mathrm{e}^{-v^{2}} \mathrm{~d} v \\
& =\frac{1}{\sqrt{\pi}} \int_{-\infty}^{\infty} \mathrm{e}^{-v^{2}} \mathrm{~d} v+O\left(\int_{\log T}^{\infty} \mathrm{e}^{-v^{2}} \mathrm{~d} v\right)+O\left(\int_{-\infty}^{-\log T} \mathrm{e}^{-v^{2}} \mathrm{~d} v\right) \\
& =1+O\left(\mathrm{e}^{-\log ^{2} T}\right),
\end{aligned}
$$

since $t-u \leqslant 0,2 T-u \geqslant 0$. Therefore, by (8.1) and the mean value theorem, we obtain

$$
\begin{aligned}
& \int_{T}^{2 T}|\zeta(\sigma+i t)|^{4} \mathrm{~d} t \leqslant \int_{T_{1}}^{T_{2}} I_{2}(t, \sigma ; G) \mathrm{d} t+O(1) \\
& =M(2 T, \sigma)-M(T, \sigma)+O(G) \\
& \quad+S(2 T+G \log T, \sigma ; G)-S(T-G \log T, \sigma ; G) \\
& \quad+R(2 T+G \log T, \sigma ; G)-R(T-G \log T, \sigma ; G) .
\end{aligned}
$$

A lower bound of a similar type for the first integral in (8.5) follows by the same procedure if we take

$$
T_{1}=T+G \log T, T_{2}=2 T-G \log T
$$

Putting together the bounds we obtain the following lemma, which is the analogue of [I2, Lemma 5.1] or [IM3, Lemma 3]. 
Lemma 11. With the notation introduced in (8.1) - (8.2) and, for $T^{1 / 3+\varepsilon} \leqslant G \leqslant$ $T^{1-\varepsilon}$, we have

$$
\begin{aligned}
& E_{2}(2 T, \sigma)-E_{2}(T, \sigma) \\
& \ll|S(2 T+G \log T, \sigma ; G)|+|S(2 T-G \log T, \sigma ; G)| \\
& \quad+|S(T+G \log T, \sigma ; G)|+|S(T-G \log T, \sigma ; G)| \\
& \quad+O(G)+O\left(T^{1 / 3} \log ^{D} T\right) .
\end{aligned}
$$

To return to the proof of (7.1)-(7.2), note that the $S$-sums can be truncated at $T G^{-1} \log T$ with a negligible error. We estimate the exponential factors trivially, and then use the Cauchy-Schwarz inequality and the bound (4.2). Thus we have

$$
\begin{aligned}
& |S(2 T+G \log T, \sigma ; G)|+|S(2 T-G \log T, \sigma ; G)| \\
& \quad+|S(T+G \log T, \sigma ; G)|+|S(T-G \log T, \sigma ; G)| \\
& \ll T^{\frac{3}{2}-2 \sigma}\left(T G^{-1} \log T\right)^{2 \sigma-\frac{1}{2}} \log ^{C} T \\
& \leqslant T G^{\frac{1}{2}-2 \sigma} \log ^{C+1} T .
\end{aligned}
$$

This gives, by Lemma 11,

$$
E_{2}(2 T, \sigma)-E_{2}(T, \sigma) \ll\left(T G^{\frac{1}{2}-2 \sigma}+G+T^{1 / 3}\right) \log ^{C} T \ll T^{\frac{2}{1+4 \sigma}} \log ^{C} T
$$

with the choice

$$
G=T^{\frac{2}{1+4 \sigma}} .
$$

From (8.7) the bound (7.2) easily follows. Note that an explicit value $C=C(\sigma)$ in Theorem 4 can also be worked out without trouble.

To prove the omega result (7.3) we argue similarly as in the case of the proof of the omega-result (see [I7], [Mo4], [Mo6])

$$
E_{2}(T)=\Omega_{ \pm}\left(T^{1 / 2}\right) .
$$

Instead of the (modified) Mellin transform

$$
\mathcal{Z}_{2}(s):=\int_{1}^{\infty}\left|\zeta\left(\frac{1}{2}+i x\right)\right|^{4} x^{-s} \mathrm{~d} x \quad(\operatorname{Re} s>1)
$$

used for the proof of (8.8), we need to use the function

$$
\mathcal{Z}_{2}(s, \tau):=\int_{1}^{\infty}|\zeta(\tau+i x)|^{4} x^{-s} \mathrm{~d} x \quad\left(\frac{1}{2}<\tau<1, \operatorname{Re} s>1\right) .
$$

The spectral decomposition of $\mathcal{Z}_{2}(s, \tau)$ is effected much in the same way as was the spectral decomposition of $\mathcal{Z}_{2}(s)$ (see [Mo4], [Mo6]). The major difference relevant 
for the omega results is that, in the case of $\mathcal{Z}_{2}(s)$ the simple poles are located at $s=\frac{1}{2} \pm i \kappa_{j}$, while in the case of $\mathcal{Z}_{2}(s, \tau)$ the simple poles are located at $s=3 / 2-2 \tau \pm i \kappa_{j}$. Hence, instead of (8.8), we obtain the omega result (7.3). In the course of the proof one needs the non-vanishing of $L_{\sigma}(\kappa)$ for infinitely many $\kappa$ (see (4.1)), which follows from (4.3) of Theorem 2. The function $\mathcal{Z}_{2}(s, \tau)$ admits meromorphic continuation over $\mathbb{C}$ where, unless $s$ lies in a neighborhood of its pole, it is of polynomial growth in $|s|$ for a fixed $\sigma$. This follows analogously as in [Mo6] and [IJM]. The crucial result is analogue of Lemma 2 of [I7], which in this case will imply that

$$
\begin{aligned}
& \int_{0}^{\infty} \int_{0}^{t} E_{2}(u, \sigma) \mathrm{d} u \cdot \mathrm{e}^{-t / T} \mathrm{~d} t \\
& \sim T^{\frac{7}{2}-2 \sigma} \operatorname{Re}\left\{\sum_{j=1}^{\infty} \alpha_{j} H_{j}^{2}\left(\frac{1}{2}\right) H_{j}\left(2 \sigma-\frac{1}{2}\right) R_{1, \sigma}\left(\kappa_{j}\right)\right\} \quad(T \rightarrow \infty),
\end{aligned}
$$

where $R_{1, \sigma}\left(\kappa_{j}\right) \ll_{\varepsilon} \exp \left(-\left(\frac{1}{2} \pi-\varepsilon\right) \kappa_{j}\right)$. From (8.10) we obtain (7.4) with the aid of ( [I7], Lemma 3). With (7.4) at our disposal, we prove easily (7.3). Let $t_{1}$ be as in (7.4). Then

$$
A t_{1}^{\frac{5}{2}-2 \sigma}<\int_{0}^{t_{1}} E_{2}(t, \sigma) \mathrm{d} t \leqslant\left(\int_{0}^{t_{1}}\left|E_{2}(t, \sigma)\right|^{a} \mathrm{~d} t\right)^{1 / a} t_{1}^{(a-1) / a}
$$

for $a>1$ by Hölder's inequality, and for $a=1$ it is trivial. In view of $T \leqslant t_{1} \leqslant B T$ we obtain

$$
A^{a} T^{1+a\left(\frac{3}{2}-2 \sigma\right)} \leqslant A^{a} t_{1}^{1+a\left(\frac{3}{2}-2 \sigma\right)} \leqslant \int_{0}^{t_{1}}\left|E_{2}(t, \sigma)\right|^{a} \mathrm{~d} t \leqslant \int_{0}^{B T}\left|E_{2}(t, \sigma)\right|^{a} \mathrm{~d} t .
$$

Changing $T$ to $T / B$ we obtain (7.3).

It remains to prove (7.5) of Theorem 5. We shall follow the proof of [Mo6, Theorem 5.3], making the necessary modifications. We wish to obtain an upper bound for $R$, the number of well-spaced points $\left\{t_{r}\right\}(r=1, \ldots, R)$ for which $E_{2}\left(t_{r}, \sigma\right) \geqslant V>0$ (the case when $E_{2}\left(t_{r}, \sigma\right) \leqslant-V$ is analogously treated, so we may consider only the former case), where

$$
\begin{gathered}
T \leqslant t_{1}<\cdots<t_{R} \leqslant 2 T, \quad t_{r+1}-t_{r} \geqslant V \log ^{-C-1} T, \\
T^{\frac{1}{4 \sigma}} \log ^{C_{2}} T \leqslant V \leqslant T^{\frac{2}{1+4 \sigma}} \log ^{C_{3}} T
\end{gathered}
$$

for suitable $C_{j}>0$. We put

$$
U=2^{-\ell} t_{r} \quad(\ell=1, \ldots, L), \quad G=V \log ^{-C_{4}} T, \quad 2^{-L} T \asymp T^{(4 \sigma+1) /(8 \sigma)},
$$


which gives

$$
E_{2}\left(t_{r}\right)=\sum_{\ell=1}^{L} \sum_{r=1}^{R}\left\{E_{2}\left(2^{1-\ell} t_{r}, \sigma\right)-E_{2}\left(2^{-\ell} t_{r}, \sigma\right)\right\}+O\left(T^{1 /(4 \sigma)} \log ^{C} T\right)
$$

by (7.2). Therefore we obtain

$$
\frac{1}{2} R V \leqslant \sum_{\ell=1}^{L} \sum_{r=1}^{R}\left\{E_{2}\left(2^{1-\ell} t_{r}, \sigma\right)-E_{2}\left(2^{-\ell} t_{r}, \sigma\right)\right\}
$$

and we now apply Lemma 11 . We may truncate each sum over $\kappa_{j}$ so that $\kappa_{j} \leqslant$ $T G^{-1} \log T$, and also expand into Taylor series the factor

$$
\exp \left(i c_{3} \kappa_{j}^{3}(U \pm G \log T)^{-2}\right)
$$

and higher power exponentials coming from (5.14), noting that the main contribution will come from the constant term, namely unity. This is important, since this procedure allows us to relax the condition $G \geqslant V^{1 / 3+\varepsilon}$ in (6.3) in such a way that $G$ and $U$ lie in a permissible range. Instead of $W(K, \ell ; z)$ (cf. [Mo6, eq. (7.2.19)]) we have $\left(\tau(r, \ell)=2^{1-\ell}+G \log T, \operatorname{Re} z=1 / \log T\right)$ now

$$
\begin{aligned}
W(K, \ell ; z) & :=\sum_{K<\kappa_{j} \leqslant 2 K} \alpha_{j} H_{j}^{2}\left(\frac{1}{2}\right)\left|H_{j}\left(2 \sigma-\frac{1}{2}\right)\right| \kappa_{j}^{2 \sigma-1}\left|\sum_{r=1}^{R} \tau(r, \ell)^{\frac{3}{2}-2 \sigma+z+i \kappa_{j}}\right| \\
& \ll K^{2 \sigma-1} \sum_{K<\kappa_{j} \leqslant 2 K} \alpha_{j} H_{j}^{2}\left(\frac{1}{2}\right)\left|H_{j}\left(2 \sigma-\frac{1}{2}\right)\right|\left|\sum_{r=1}^{R} \tau(r, \ell)^{\frac{3}{2}-2 \sigma+z+i \kappa_{j}}\right| .
\end{aligned}
$$

To the sum over $\kappa_{j}$ we apply the Cauchy-Schwarz inequality, noting that for $\sigma>\frac{1}{2}$

$$
\begin{aligned}
& \sum_{K<\kappa_{j} \leqslant 2 K} \alpha_{j}\left|H_{j}\left(\frac{1}{2}\right) H_{j}\left(2 \sigma-\frac{1}{2}\right)\right|^{2} \\
\leqslant & \left(\sum_{K<\kappa_{j} \leqslant 2 K} \alpha_{j} H_{j}^{4}\left(\frac{1}{2}\right) \sum_{K<\kappa_{j} \leqslant 2 K} \alpha_{j} H_{j}^{4}\left(2 \sigma-\frac{1}{2}\right)\right)^{1 / 2} \\
\ll & K^{2} \log ^{C} K,
\end{aligned}
$$

since both sums above are bounded by $K^{2} \log ^{C} K$. For the sum with $H_{j}^{4}\left(\frac{1}{2}\right)$ this is [Mo6, Theorem 3.4], and the other sum is treated analogously. This yields

$$
W^{2}(K, \ell ; z) \ll K^{4 \sigma} \log ^{C} K \sum_{K<\kappa_{j} \leqslant 2 K} \alpha_{j} H_{j}^{2}\left(\frac{1}{2}\right)\left|\sum_{r=1}^{R} \tau(r, \ell)^{\frac{3}{2}-2 \sigma+z+i \kappa_{j}}\right|^{2} .
$$


With (8.13) we obtain, on applying [Mo6, eq. (5.6.3)] (this is a variant of the spectral large sieve), the uniform bound

$$
W^{2}(K, \ell ; z) \ll K^{4 \sigma+1} \log ^{C} K\left(K+T V^{-1}\right) R T^{3-4 \sigma} 2^{-\ell} .
$$

With the aid of (8.12) this yields, similarly as in [Mo6, Chapter 5]

$$
\begin{aligned}
R V & \ll \max _{K \leqslant T G^{-1} \log T} K^{-3 / 2}\left(K^{2 \sigma+1}+T^{1 / 2} V^{-1 / 2} K^{2 \sigma+\frac{1}{2}}\right) R^{1 / 2} T^{3 / 2-2 \sigma} \log ^{C} T \\
& \ll R^{1 / 2} T V^{\frac{1}{2}-2 \sigma} \log ^{C} T .
\end{aligned}
$$

Therefore we obtain

$$
R \ll T^{2} V^{-1-4 \sigma} \log ^{C} T,
$$

which easily leads to (7.2). The part where $\left|E_{2}(t, \sigma)\right| \leqslant T^{1 /(4 \sigma)} \log ^{C} T$ is trivial, so we may restrict integration to the set $\mathcal{S}$, where $\left|E_{2}(t, \sigma)\right| \geqslant T^{1 /(4 \sigma)} \log ^{C} T$. Consider the subset $\mathcal{S}_{V}$ of $\mathcal{S}$, where $V \leqslant\left|E_{2}(t, \sigma)\right|<2 V, t \in \mathcal{S} \cap\left[\frac{1}{2} T, T\right]$. We divide the interval $\left[\frac{1}{2} T, T\right]$ into subintervals of length $V \log ^{C} T$, allowing the end subintervals to be possibly shorter. Then the number $R=R_{V}$ of those subintervals (considering separately subintervals with even and odd indices) which contain a point from $\mathcal{S}_{V}$ is bounded by (8.14). Hence we have

$$
\int_{\mathcal{S}_{V}}\left|E_{2}(t, \sigma)\right|^{4 \sigma} \mathrm{d} t \ll R_{V} V \log ^{C} T V^{4 \sigma} \ll T^{2} \log ^{C} T,
$$

and since there are $O(\log T)$ choices for $V$, we have

$$
\int_{\frac{1}{2} T}^{T}\left|E_{2}(t, \sigma)\right|^{4 \sigma} \mathrm{d} t \ll T^{2} \log ^{C} T .
$$

Replacing $T$ by $T 2^{-j}$ and summing the above bounds over $j \in \mathbb{N}$ we obtain (7.5). The proof of Theorem 5 is complete.

\section{References}

[BM] R.W. Bruggeman and Y. Motohashi, 'A new approach to the spectral theory of the fourth moment of the Riemann zeta-function', Journal reine angew. Math. $\mathbf{5 7 9}$ (2005), 75-114.

[I1] A. Ivić, 'The Riemann zeta-function', John Wiley and Sons, New York, 1985 (2nd ed., Dover, 2003).

[I2] A. Ivić, 'Mean values of the Riemann zeta-function', LN's 82, Tata Institute of Fundamental Research, Bombay, 1991 (distr. by Springer Verlag, Berlin etc.). 
[I3] A. Ivić, 'The moments of the zeta-function on the line $\sigma=1$ ', Nagoya Math. J. 135 (1994), 113-120.

[I4] A. Ivić, 'On the fourth moment of the Riemann zeta-function', Publs. Inst. Math. (Belgrade) 57(71) (1995), 101-110.

[I5] A. Ivić, 'The Mellin transform and the Riemann zeta-function', in "Proceedings of the Conference on Elementary and Analytic Number Theory (Vienna, July 18-20, 1996)", Universität Wien \& Universität für Bodenkultur, Eds. W.G. Nowak and J. Schoißengeier, Vienna 1996, 112-127.

[I6] A. Ivić, Some problems on mean values of the Riemann zeta-function, Journal de Théorie des Nombres Bordeaux 8 (1996), 101-122.

[I7] A. Ivić, 'On the error term for the fourth moment of the Riemann zeta-function', J. London Math. Soc. 60 (2)(1999), 21-32.

[I8] A. Ivić, 'On the integral of the error term in the fourth moment of the Riemann zeta-function', Functiones et Approximatio 28 (2000), 37-48.

[I9] A. Ivić, 'On the moments of Hecke series at central points', Functiones et Approximatio 30 (2002), 49-82.

[IM1] A. Ivić and Y. Motohashi, 'A note on the mean value of the zeta and L-functions VII', Proc. Japan Acad. Ser. A 66 (1990), 150-152.

[IM2] A. Ivić and Y. Motohashi, 'The mean square of the error term for the fourth moment of the zeta-function', Proc. London Math. Soc. (3) 66 (1994), 309-329.

[IM3] A. Ivić and Y. Motohashi, 'The fourth moment of the Riemann zeta-function', J. Number Theory $5 \mathbf{1}$ (1995), 16-45.

[IJM] A. Ivić, M. Jutila and Y. Motohashi, 'The Mellin transform of powers of the zeta-function', Acta Arith. 95 (2000), 305-342.

[K1] A. Kačènas, 'Mean values of the Riemann zeta-function in the critical strip', Doctoral Thesis, Vilnius University, Vilnius, 1996.

[K2] A. Kačenas, 'Asymptotics of the fourth power moment of the Riemann zeta-function in the critical strip', Lithuanian Math. J. 36 (1996), 32-44.

[KS] S. Katok and P. Sarnak, 'Heegner points, cycles and Maass forms', Israel J. Math. 84 (1993), 193-227.

[L] N.N. Lebedev, 'Special functions and their applications', Dover Publications, Inc., New York, 1972.

[Ma] K. Matsumoto, 'Recent developments in the mean square theory of the Riemann zeta and allied functions', in "Number Theory", eds. R.P. Bambah et al., Hindustan Book Agency \& Indian National Science Academy, Birkhäuser, 2000, pp. 241-286.

[Mo1] Y. Motohashi, 'Spectral mean values of Maass wave form $L$-functions', $J$. Number Theory 42 (1992), 258-284.

[Mo2] Y. Motohashi, 'An explicit formula for the fourth power mean of the Riemann zeta-function', Acta Math. 170 (1993), 181-220. 
[Mo3] Y. Motohashi, 'The mean square of Hecke $L$-functions attached to holomorphic cusp forms', Kokyuroku Res. Inst. Math. Kyoto Univ. 886 (1994), 214-227.

[Mo4] Y. Motohashi, 'A relation between the Riemann zeta-function and the hyperbolic Laplacian', Annali Scuola Norm. Sup. Pisa, Cl. Sci. IV ser. 22 (1995), 299-313.

[Mo5] Y. Motohashi, 'The Riemann zeta-function and the non-Euclidean Laplacian', Sugaku Expositions, AMS 8 (1995), 59-87.

[Mo6] Y. Motohashi, 'Spectral theory of the Riemann zeta-function', Cambridge University Press, Cambridge, 1997.

[Mo7] Y. Motohashi, 'A note on the mean value of the zeta and $L$-functions. XIV', Proc. Japan Acad. 80A (2004), 28-33.

[WW] E.T. Whittaker and G.N. Watson, 'A Course of Modern Analysis', Cambridge University Press (4th. ed.), London, 1963.

Addresses: Aleksandar Ivić, Katedra Matematike RGF-a, Universiteta u Beogradu, Djušina 7, 11000 Beograd, Serbia and Montenegro;

Yoichi Motohashi, Department of Mathematics, College of Science and Technology, Nihon University, Surugadai, Tokyo 101-8308, Japan

E-mail: ivic@rgf.bg.ac.yu; ymoto@math.cst.nihon-u.ac.jp

Received: 5 October 2004; revised: 8 February 2006 